\title{
The Electronic Structure of Mn in Oxides, Coordination Complexes, and the Oxygen-Evolving Complex of Photosystem II Studied by Resonant Inelastic X-ray Scattering
}

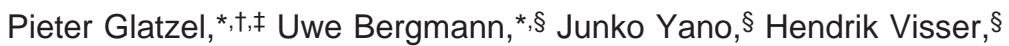 \\ John H. Robblee, $§$ Weiwei Gu, ${ }^{\dagger}$ Frank M. F. de Groot, $¥$ George Christou," \\ Vincent L. Pecoraro, ${ }^{\perp}$ Stephen P. Cramer, ${ }^{*, \S, \dagger}$ and Vittal K. Yachandra ${ }^{*, \S}$ \\ Contribution from the Department of Applied Science, University of California, \\ Davis, California 95616, Department of Inorganic Chemistry and Catalysis, Debye Institute, \\ Utrecht University, 3584 CA Utrecht, The Netherlands, Department of Chemistry, University of \\ Florida, Gainesville, Florida 32611, Department of Chemistry, University of Michigan, \\ Ann Arbor, Michigan 48109-1055, and Melvin Calvin Laboratory, Physical Biosciences \\ Division, Lawrence Berkeley National Laboratory, Berkeley, California 94720
}

Received September 18, 2003; E-mail: Pieter.Glatzel@gmx.org; VKYachandra@lbl.gov

\begin{abstract}
Resonant inelastic X-ray scattering (RIXS) was used to collect $\mathrm{Mn} \mathrm{K}$ pre-edge spectra and to study the electronic structure in oxides, molecular coordination complexes, as well as the $S_{1}$ and $S_{2}$ states of the oxygen-evolving complex (OEC) of photosystem II (PS II). The RIXS data yield two-dimensional plots that can be interpreted along the incident (absorption) energy or the energy transfer axis. The second energy dimension separates the pre-edge (predominantly $1 \mathrm{~s}$ to $3 \mathrm{~d}$ transitions) from the main K-edge, and a detailed analysis is thus possible. The 1s2p RIXS final-state electron configuration along the energy transfer axis is identical to conventional L-edge absorption spectroscopy, and the RIXS spectra are therefore sensitive to the Mn spin state. This new technique thus yields information on the electronic structure that is not accessible in conventional K-edge absorption spectroscopy. The line splittings can be understood within a ligand field multiplet model, i.e., (3d,3d) and (2p,3d) two-electron interactions are crucial to describe the spectral shapes in all systems. We propose to explain the shift of the $\mathrm{K}$ pre-edge absorption energy upon $\mathrm{Mn}$ oxidation in terms of the effective number of $3 \mathrm{~d}$ electrons (fractional $3 \mathrm{~d}$ orbital population). The spectral changes in the Mn $1 \mathrm{~s} 2 \mathrm{p}_{3 / 2}$ RIXS spectra between the PS II $\mathrm{S}_{1}$ and $\mathrm{S}_{2}$ states are small compared to that of the oxides and two of the coordination complexes $\left(\mathrm{Mn}^{\mathrm{III}}(\mathrm{acac})_{3}\right.$ and $\left.\mathrm{Mn}^{\mathrm{IV}}(\mathrm{sal})_{2}(\mathrm{bipy})\right)$. We conclude that the electron in the step from $S_{1}$ to $S_{2}$ is transferred from a strongly delocalized orbital.
\end{abstract}

\section{Introduction}

Photosynthetic water oxidation sustains most life on earth by providing the oxygen we breathe and metabolize. The reaction $2 \mathrm{H}_{2} \mathrm{O} \rightarrow \mathrm{O}_{2}+4 \mathrm{H}^{+}+4 \mathrm{e}^{-}$is catalyzed in the oxygenevolving complex (OEC) of the multisubunit protein/chlorophyll photosystem II (PS II) complex. ${ }^{1,2}$ The OEC contains four Mn atoms that are at the active site for catalytic water oxidation. The oxidation states II, III, and IV of Mn are energetically available, and $\mathrm{Mn}$ is thus well-suited for redox reactions where several oxidizing equivalents are exchanged. Manganese therefore provides the OEC with a high degree of redox and chemical flexibility.

\footnotetext{
$\dagger$ University of California at Davis.

$\doteqdot$ Utrecht University.

$\S$ Lawrence Berkeley National Laboratory.

"University of Florida.

${ }^{\perp}$ University of Michigan.

(1) Britt, R. D. In Oxygenic Photosynthesis: The Light Reactions; Ort, D. R., Yocum, C. F., Eds.; Kluwer Academics Publishers: Dordrecht, The Netherlands, 1996; Vol. 4, pp 137-164.

(2) Diner, B. A.; Babcock, G. T. In Oxygenic Photosynthesis: The Light Reactions; Ort, D. R.; Yocum, C. F., Eds.; Kluwer Academics Publishers: Dordrecht, The Netherlands, 1996; Vol. 4, pp 213-247.
}

The OEC cycles through five intermediate states called S-states $\left(\mathrm{S}_{i}, i=0-4\right)$ during water oxidation. ${ }^{3,4}$ The general understanding is that the electrons are released in the OEC either from $\mathrm{Mn}$ or, as has been proposed for the $S_{2}-S_{3}$ transition, from a Mn ligand. ${ }^{5}$ Within this picture of a localized oxidation, Mn oxidation states are assigned to each $\mathrm{S}$ state. A widely held model for the Mn oxidation states in $\mathrm{S}_{1}$ and $\mathrm{S}_{2}$ is $\mathrm{Mn}_{4}\left(\mathrm{III}_{2}, \mathrm{IV}_{2}\right)$ and $\mathrm{Mn}_{4}\left(\mathrm{III}, \mathrm{IV}_{3}\right)$, respectively; i.e., a $\mathrm{Mn}(\mathrm{III})$ to $\mathrm{Mn}(\mathrm{IV})$ oxidation occurs in the $S_{1} \rightarrow S_{2}$ transition. ${ }^{6-8}$ We show in this article that the electron in the $S_{1} \rightarrow S_{2}$ transition is removed from a strongly delocalized orbital and that it can therefore not be assigned to just one element in the OEC.

(3) Joliot, B.; Barbieri, G.; Chabaud, R. Photochem. Photobiol. 1969, 10, 309329.

(4) Kok, B.; Forbush, B.; McGloin, M. Photochem. Photobiol. 1970, 11, 457475 .

(5) Messinger, J.; Robblee, J. H.; Bergmann, U.; Fernandez, C.; Glatzel, P. Visser, H.; Cinco, R. M.; McFarlane, K. L.; Bellacchio, E.; Pizarro, S. A. Cramer, S. P.; Sauer, K.; Klein, M. P.; Yachandra, V. K. J. Am. Chem. Soc. 2001, 123, 7804-7820.

(6) Carrell, T. G.; Tyryshkin, A. M.; Dismukes, G. C. J. Biol. Inorg. Chem. 2002, 7, 2-22.

(7) Siegbahn, P. E. M. Curr. Opin. Chem. Biol. 2002, 6, 227-235.

(8) Yachandra, V. K.; Sauer, K.; Klein, M. P. Chem. Rev. 1996, 96, 29272950 
The electronic structure of the OEC cluster has been studied by many spectroscopic methods ranging from magnetic resonance techniques (EPR, NMR, ENDOR) to X-ray absorption and fluorescence spectroscopy. ${ }^{5,8-11} \mathrm{X}$-ray spectroscopies involve excitations of core level electrons and therefore have the advantage of being element-specific. It is thus possible to attempt to identify the change of electronic structure with an element in the OEC during the water oxidation process. The near-edge structure (XANES) of the Mn K-edge absorption spectrum mainly reflects the $\mathrm{Mn}$ p-orbital contribution to the unoccupied molecular orbitals due to the dipole selection rules. ${ }^{12} \mathrm{Mn}$ XANES spectra have been used to monitor changes in the Mn oxidation state in the different S-states. ${ }^{8,13}$ Instead of measuring the X-ray absorption, it is possible to record the $\mathrm{K} \beta$ fluorescence emission that occurs when a $1 \mathrm{~s}$ core hole is filled by an electron from the $3 \mathrm{p}$ shell..$^{5,14-16}$

Interpretation of XANES is difficult because of the many factors that influence the spectral shape. ${ }^{17,18} \mathrm{~K} \beta$ spectra show rather small chemical shifts, and they require ionization of the Mn 1s shell. Both inner-shell spectroscopies thus have their limitations, and alternative approaches are desirable. A weak spectral feature arises at incident photon energies lower than the main $\mathrm{K}$ absorption edge. The potential of this $\mathrm{K}$ absorption pre-edge structure to extract information on the metal atom electronic structure and the local symmetry at the metal site is well-known. ${ }^{19-23}$ However, the K pre-edge spectral features are usually weak compared to the main edge because they mostly draw their intensities from quadrupole transition matrix elements. Furthermore, the energy separation between pre-edge and main edge decreases toward the early transition metals. A detailed analysis of the K pre-edge spectral features in conventional absorption spectroscopy of early transition metals is therefore often limited and associated with a rather large uncertainty because a strong background from dipole-allowed transition at higher energies has to be subtracted. Hence, only few studies are available for Mn. ${ }^{24-29} \mathrm{We}$ show in this article that resonant inelastic X-ray scattering (RIXS) spectroscopy can

(9) Britt, R. D.; Peloquin, J. M.; Campbell, K. A. Annu. Rev. Biophys. Biomol. Struct. 2000, 29, 463-495.

(10) Sharp, R. R. In Manganese Redox Enzymes; Pecoraro, V. L., Eds.; VCH Publishers: New York, 1992; pp 177-196.

(11) Bergmann, U.; Grush, M. M.; Horne, C. R.; DeMarois, P.; Penner-Hahn, J. E.; Yocum, C. F.; Wright, D. W.; Dube, C. E.; Armstrong, W. H.; Christou, G.; Eppley, H. J.; Cramer, S. P. J. Phys. Chem. B 1998, 102, $8350-8352$

(12) X-ray Absorption: Principles, Applications, Techniques of EXAFS, SEXAFS, and XANES; Koningsberger, D. C., Prins, R., Eds.; Wiley: New York, 1988.

(13) Penner-Hahn, J. E.; Fronko, R. M.; Pecoraro, V. L.; Yocum, C. F.; Betts, S. D.; Bowlby, N. R. J. Am. Chem. Soc. 1990, 112, 2549-2557.

(14) Peng, G.; Degroot, F. M. F.; Hämäläinen, K.; Moore, J. A.; Wang, X.; Grush, M. M.; Hastings, J. B.; Siddons, D. P.; Armstrong, W. H.; Mullins, O. C.; Cramer, S. P. J. Am. Chem. Soc. 1994, 116, 2914-2920.

(15) Glatzel, P. X-ray Fluorescence Emission Following K Capture and 1 s Photoionization of $\mathrm{Mn}$ and $\mathrm{Fe}$ in Various Chemical Environments. Ph.D. Thesis, Hamburg University, Hamburg, Germany, 2001.

(16) Glatzel, P.; Bergmann, U.; de Groot, F. M. F.; Cramer, S. P. Phys. Rev. B 2001, 64, 045109

(17) Rehr, J. J.; Albers, R. C. Rev. Mod Phys. 2000, 72, 621-654.

(18) de Vries, A. H.; Hozoi, L.; Broer, R. Int. J. Quantum Chem. 2003, 91 57-61

(19) Westre, T. E.; Kennepohl, P.; DeWitt, J. G.; Hedman, B.; Hodgson, K. O.; Solomon, E. I. J. Am. Chem. Soc. 1997, 119, 6297-6314.

(20) Wilke, M.; Farges, F.; Petit, P. E.; Brown, G. E.; Martin, F. Am. Mineral. 2001, 86, 714-730.

(21) Farges, F.; Brown, G. E.; Petit, P. E.; Munoz, M. Geochim. Cosmochim. Acta 2001, 65, 1665-1678.

(22) Pellegrin, E.; Fink, J.; Chen, C. T.; Xiong, Q.; Lin, Q. M.; Chu, C. W. Phys. Rev. B 1996, 53, 2767-2772.

(23) Farges, F.; Brown, G. E.; Rehr, J. J. Phys. Rev, B 1997, 56, 1809-1819.

(24) Nietubyc, R.; Sobczak, E.; Attenkofer, K. E. J. Alloys Compd 2001, 328, $126-131$ be used to separate the pre-edge structure from the main $\mathrm{K}$ absorption edge.

The pre-edge arises from excitations into the lowest unoccupied states that are partly formed by Mn 3d orbitals. Spectral shape and energy position ("center-of-gravity") are influenced by the symmetry in which the metal atom is embedded as well as the effective number of electrons in the metal $3 \mathrm{~d}$ shell (fractional 3d-orbital population). This number does not coincide with the formal number as derived from the oxidation state in many transition-metal systems because of metal-ligand covalency. ${ }^{30,31}$ It can be obtained by means of a population analysis where a molecular orbital is expanded in terms of atomic orbitals and the fractional population of each atomic orbital is determined. ${ }^{32,33}$ Particularly in core hole spectroscopy, with its local probing of the electronic structure, the concept of metal oxidation state to explain spectral shapes is only of limited merit and a different picture has to be adopted.

The model systems that we studied are the Mn oxides $\mathrm{MnO}$, $\mathrm{Mn}_{3} \mathrm{O}_{4}, \mathrm{Mn}_{2} \mathrm{O}_{3}$, and $\mathrm{MnO}_{2}$ and the mononuclear coordination complexes $\mathrm{Mn}^{\mathrm{II}}(\mathrm{acac})_{2}\left(\mathrm{H}_{2} \mathrm{O}\right)_{2}, \mathrm{Mn}^{\mathrm{III}}(\mathrm{acac})_{3},\left[\mathrm{Mn}^{\mathrm{III}}(5-\mathrm{Cl}-\mathrm{Salpn})-\right.$ $\left.\left(\mathrm{CH}_{3} \mathrm{OH}\right)_{2}\right]\left(\mathrm{O}_{3} \mathrm{SCF}_{3}\right)$, and $\mathrm{Mn}^{\mathrm{IV}}(\mathrm{sal})_{2}$ (bipy) (acac, acetylacetonate; $\mathrm{H}_{2}$ (5-Cl-Salpn), 1,3-bis(5-chlorosalicylideneiminato)propane; bipy, bipyridine; and sal, salicylate). The local symmetry at the Mn site can be approximated in all oxides by either octahedral $\left(O_{h}\right)$ or tetragonal $\left(D_{4 h}\right)$ symmetry, with the exception of $\mathrm{Mn}_{3} \mathrm{O}_{4}$, which exhibits a spinel structure $\mathrm{Mn}^{\mathrm{II}} \mathrm{Mn}^{\mathrm{III}} \mathrm{Mn}^{\mathrm{III}} \mathrm{O}_{4}$ with one $\mathrm{Mn}(\mathrm{III})$ in tetrahedral coordination. ${ }^{34}$ Manganese in the coordination complexes is six-coordinated with only oxygen ligands in the acetylacetonate samples and four oxygen and two nitrogen ligands in $\mathrm{Mn}^{\mathrm{IV}}$ (sal) $)_{2}$ (bipy) and $\left[\mathrm{Mn}^{\mathrm{III}}(5-\mathrm{Cl}-\mathrm{Salpn})\left(\mathrm{CH}_{3}-\right.\right.$ $\left.\mathrm{OH})_{2}\right]\left(\mathrm{O}_{3} \mathrm{SCF}_{3}\right)$. The $\mathrm{Mn}$ model compounds can be divided into two groups: The Mn oxides with a pronounced band structure formation and molecular Mn coordination complexes. We first present and discuss the results of the two groups of model compounds separately because of their different solid-state structures. We then interpret the results in a unified picture.

1s2p RIXS Spectroscopy. In the present study, we use RIXS to isolate the $\mathrm{K}$ pre-edge spectral features from the main absorption edge. The spectra can furthermore be interpreted in terms of a second energy axis, the energy transfer axis. The RIXS technique is outlined below. A more comprehensive discussion will be published elsewhere. ${ }^{35}$

The electronic states that give rise to the edge of an absorption spectrum are resonantly excited states that subsequently decay

(25) Schulze, D. G.; Sutton, S. R.; Bajt, S. Soil Sci. Soc. Am. J. 1995, 59, 15401548 .

(26) Kusunoki, M.; Ono, T.; Matsushita, T.; Oyanagi, H.; Inoue, Y. J. Biochem. 1990, 108, 560-567.

(27) Visser, H.; Anxolabehere-Mallart, E.; Bergmann, U.; Glatzel, P.; Robblee, J. H.; Cramer, S. P.; Girerd, J. J.; Sauer, K.; Klein, M. P.; Yachandra, V. K. J. Am. Chem. Soc. 2001, 123, 7031-7039.

(28) Gilbert, B.; Frazer, B. H.; Belz, A.; Conrad, P. G.; Nealson, K. H.; Haskel, D.; Lang, J. C.; Srajer, G.; De Stasio, G. J. Phys. Chem. A 2003, 107, 2839-2847

(29) Shoji, H.; Taguchi, M.; Hirai, E.; Iwazumi, T.; Kotani, A.; Nanao, S.; Isozumi, Y. J. Phys. Soc. Jpn. 2003, 72, 1560-1569.

(30) Kawai, J.; Suzuki, C.; Adachi, H.; Konishi, T.; Gohshi, Y. Phys. Rev. B 1994, 50, 11347-54.

(31) Zampieri, G.; Abbate, M.; Prado, F.; Caneiro, A.; Morikawa, E. Physica B 2002, 320, 51-55.

(32) Mulliken, R. S. J. Chem. Phys. 1955, 23, 1833.

(33) Wasinger, E. C.; de Groot, F. M. F.; Hedman, B.; Hodgson, K. O.; Solomon, E. I. J. Am. Chem. Soc. 2003, 125, 12894-12906.

(34) Wells, A. F. Structural Inorganic Chemistry, 5th ed., repr. with corrections; Clarendon Press: New York, 1990

(35) Glatzel, P.; Bergmann, U. Coord. Chem. Rev. 2004, in press. 
Ground State

Intermediate States

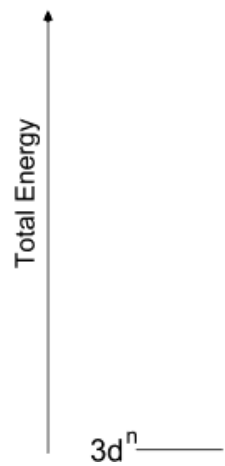

Figure 1. Simplified RIXS energy scheme for $1 s 2 p$ RIXS in a transitionmetal ion. The vertical axis indicates the total energy of the electron configuration. For simplicity, atomic configurations are used and only 1s to $3 \mathrm{~d}$ excitations are shown. The discrete resonances with $1 \mathrm{~s} 3 \mathrm{~d}^{n+1}$ configurations form the K pre-edge. The RIXS final-state electron configuration can also be reached by a soft X-ray $2 \mathrm{p}$ to $3 \mathrm{~d}$ absorption process.

(Figure 1). In the case of a $3 \mathrm{~d}$ transition-metal ion, the radiative decay with the highest probability after 1 s core hole creation is a $2 \mathrm{p}$ to $1 \mathrm{~s}$ transition. ${ }^{36}$ The spectroscopy is therefore denoted $1 \mathrm{~s} 2 \mathrm{p}$ RIXS to describe the core hole in the intermediate (1s) and final (2p) state. The fluorescence light emitted during the decay process can be recorded using a secondary crystal analyzer with an energy bandwidth similar to that of the primary monochromator of the synchrotron beamline. ${ }^{37-39}$ The process can be viewed as an inelastic scattering of the incident photon at the $\mathrm{Mn}$ atom and is theoretically described by the KramersHeisenberg formula: ${ }^{40,41}$

$$
\begin{aligned}
& F(\Omega, \omega)= \\
& \sum_{f}\left|\sum_{n} \frac{\left\langle f\left|T_{2}\right| n\right\rangle\left\langle n\left|T_{1}\right| g\right\rangle}{E_{g}-E_{n}+\Omega-i\left(\Gamma_{K} / 2\right)}\right|^{2} * \frac{\Gamma_{\mathrm{L}} / 2 \pi}{\left(E_{g}-E_{f}+\Omega-\omega\right)^{2}+\Gamma_{\mathrm{L}}^{2} / 4}
\end{aligned}
$$

This description is analogous to optical resonance Raman spectroscopy. ${ }^{42,43}$ The intermediate state $|n\rangle$ is reached from the ground state $|g\rangle$ via a transition operator $T_{1}$. In a simplified picture using atomic configuration, we can write $|g\rangle=3 \mathrm{~d}^{n}$ and $|n\rangle=1 \mathrm{~s} 3 \mathrm{~d}^{n+1}$, i.e., a $1 \mathrm{~s}$ electron is resonantly excited into a $3 \mathrm{~d}$ orbital. The intermediate states $|n\rangle$ in RIXS spectroscopy are the final states in conventional K-edge absorption spectroscopy. $T_{1}$ identifies with the quadrupole transition operator if the scattering atom is six-coordinated with identical ligands in an octahedral geometry ( $O_{h}$ symmetry). The K pre-edge can obtain some contribution from dipole transitions if the local symmetry is lower than $O_{h}{ }^{44-46}$ We note that a Jahn-Teller distortion of

(36) Kortright, J. B.; Thompson, A. C. X-ray Data Booklet, 2nd ed.; Lawrence Berkeley National Laboratory: Berkeley, CA, 2001.

(37) Stojanoff, V.; Hamalainen, K.; Siddons, D. P.; Hastings, J. B.; Berman, L. E.; Cramer, S.; Smith, G. Rev. Sci. Instrum. 1992, 63, 1125-1127.

(38) Bergmann, U.; Cramer, S. P. Proc. SPIE Int. Soc. Opt. Eng. 1998, 3448, $198-209$.

(39) Schülke, W.; Kaprolat, A.; Fischer, T.; Hoppner, K.; Wohlert, F. Rev. Sci. Instrum. 1995, 66, 2446-2452.

(40) Kotani, A.; Shin, S. Rev. Mod. Phys. 2001, 73, 203-46.

(41) Gel'mukhanov, F.; Agren, H. Phys. Rep.-Rev. Sec. Phys. Lett. 1999, 312 91-330

(42) Solomon, E. I.; Hanson, M. A. In Inorganic Electronic Structure and Spectroscopy; Solomon, E. I., Lever, A. B. P., Eds.; John-Wiley \& Sons: New York, 1999; Vol. 2, pp 1-129.

(43) Czernuszewicz, R. S.; Spiro, T. G. In Inorganic Electronic Structure and Spectroscopy; Solomon, E. I.; Lever, A. B. P., Eds.; Wiley \& Sons: New York, 1999; Vol. 1, pp 353-441.
$O_{h}$ to $D_{4 h}$ symmetry does not introduce a dipole contribution to $T_{1}$ because inversion symmetry still holds.

The final states are reached via a $2 \mathrm{p}$ to $1 \mathrm{~s}$ transition, and $T_{2}$ therefore identifies with the dipole operator. The 1s $2 p$ RIXS final-state configuration $|f\rangle=2 \mathrm{p}^{5} 3 \mathrm{~d}^{n+1}$ is identical to the finalstate configuration in soft X-ray L-edge absorption spectroscopy. Transition-metal L-edge spectra with their pronounced chemical sensitivity have been discussed in depth by numerous authors. ${ }^{28,47-49}$ In particular, it was found that the strong $(2 \mathrm{p}, 3 \mathrm{~d})$ multiplet interaction makes the L-edge more sensitive to the metal spin state. The same interaction also occurs in the $1 \mathrm{~s} 2 \mathrm{p}$ RIXS final states. In a recent RIXS study of a series of $\mathrm{Ni}$ coordination complexes, similar spectral shapes as in L-edge spectroscopy were found in the energy transfer direction of the RIXS data, thus demonstrating that RIXS is also sensitive to the metal spin state..$^{50}$

The $2 \mathrm{p}^{5} 3 \mathrm{~d}^{n+1}$ final states are generally divided into the $\mathrm{L}_{3}$ and the $\mathrm{L}_{2}$ lines that are split by the $2 \mathrm{p}$ spin-orbit interaction with total angular momenta of $j=3 / 2\left(2 \mathrm{p}_{3 / 2}\right)$ and $j=1 / 2\left(2 \mathrm{p}_{1 / 2}\right)$, respectively. We will focus our analysis of the experimental results in this article on the $\mathrm{L}_{3}$ final states because a strong background arises in the range of the $\mathrm{L}_{2}$ line that introduces a large uncertainty into the analysis. The background is due to intense dipole-allowed transitions at higher incident energies. ${ }^{35}$

The incident energy $\Omega$ as well as the emitted energy $\omega$ are varied in a RIXS experiment. The recorded intensity is proportional to $\mathrm{F}(\Omega, \omega)$ (cf. eq 1 ) and is thus plotted versus a two-dimensional grid. To assign the total energy of an electronic state to the axes of the contour plots, we will use the energy transfer or final-state energy $\Omega-\omega$ as opposed to the emitted energy $\omega$ (cf. Figure 1). The energy transfer axis relates to the excitation energy in L-edge absorption spectroscopy. The Kramers-Heisenberg equation contains two Lorentzian line shapes for the incident energy $\Omega$ and the energy transfer $\Omega-\omega$. The lifetime broadenings $\Gamma_{\mathrm{K}}$ for the intermediate states and $\Gamma_{\mathrm{L}}$ for the final states thus apply in the $\Omega$ and $\Omega-\omega$ direction, respectively. An experimental spectrum is further broadened by the energy bandwidths of the incident energy monochromator and the crystal analyzer.

We illustrate some aspects of RIXS spectroscopy in the following by discussing a theoretical RIXS plane for a model system with the energy scheme in Figure 1 . We use $\Gamma_{K}=1.1$ $\mathrm{eV}$ and $\Gamma_{\mathrm{L}}=0.5 \mathrm{eV}$ full width at half-maximum (fwhm) for the Mn lifetime broadenings. ${ }^{51}$ The RIXS spectrum can be shown as a surface plot (Figure 2) or a contour plot (Figure 3 bottom left). For the purpose of this study where we analyze the spectra with respect to energy shifts and line splittings, it is more instructive to use the contour plots, and we will do so when discussing the experimental results.

(44) Figgis, B. N. Introduction to Ligand Fields; Interscience: New York, 1967. (45) Griffith, J. S. The Theory of Transition-Metal Ions; University Press: Cambridge, U.K., 1964.

(46) Ballhausen, C. J.; Gray, H. B. Molecular Orbital Theory; W. A Benjamin: New York, 1964.

(47) deGroot, F. M. F. J. Electron Spectrosc. Relat. Phenom. 1994, 67, 529622.

(48) Cramer, S. P.; Degroot, F. M. F.; Ma, Y.; Chen, C. T.; Sette, F.; Kipke, C. A.; Eichhorn, D. M.; Chan, M. K.; Armstrong, W. H.; Libby, E.; Christou, G.; Brooker, S.; Mckee, V.; Mullins, O. C.; Fuggle, J. C. J. Am. Chem. Soc. 1991, 113, 7937-7940.

(49) Kurata, H.; Colliex, C. Phys. Rev. B 1993, 48, 2102-2108.

(50) Glatzel, P.; Bergmann, U.; Gu, W. W.; Wang, H. X.; Stepanov, S ; Mandimutsira, B. S.; Riordan, C. G.; Horwitz, C. P.; Collins, T.; Cramer, S. P. J. Am. Chem. Soc. 2002, 124, 9668-9669.

(51) Krause, M. O.; Oliver, J. H. J. Phys. Chem. Ref. Data 1979, 8, 329-337. 


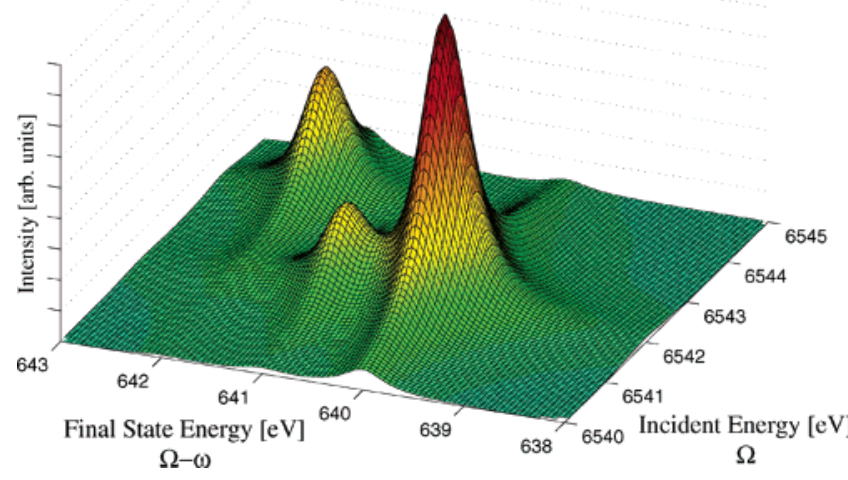

Figure 2. Theoretical RIXS spectrum displayed as a surface plot based on the energy scheme in Figure 1. Continuum excitations are neglected, and only the discrete resonances are shown.

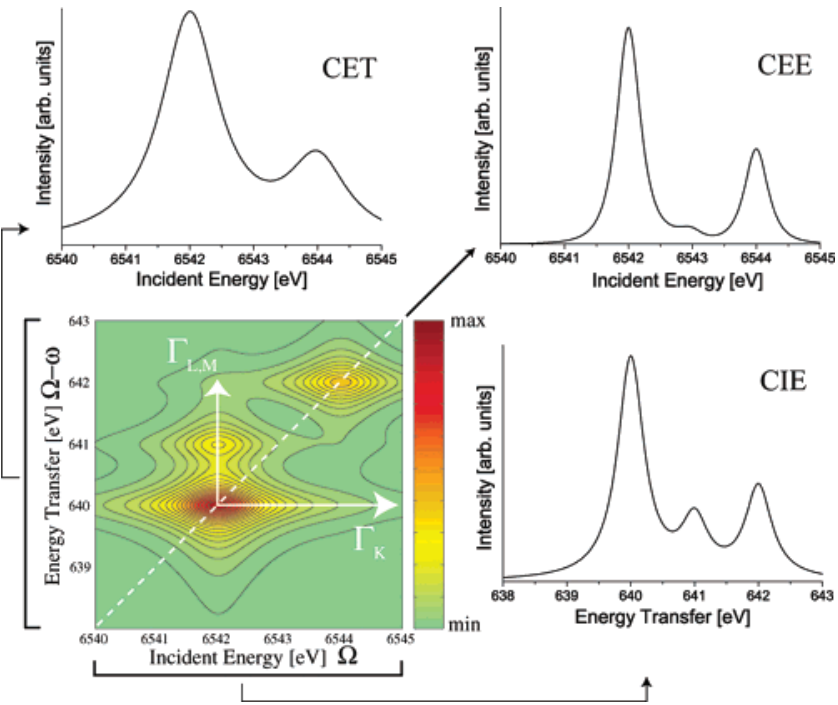

Figure 3. Theoretical RIXS spectrum displayed as a contour plot based on the energy scheme in Figure 1. The relative spectral intensities in the RIXS plane are given in the color bar. Three line plots were generated from the RIXS spectrum. The lifetime broadenings and the diagonal cut that results in the constant emission energy (CEE) line plot are indicated in the RIXS plane. The peak that lies off the diagonal corresponds to the multiplet split final state that is shown as a dotted line in Figure 1. The square brackets indicate the integration range for the constant energy transfer (CET) and constant incident energy (CIE) line plots, respectively.

The example in Figure 3 shows how the lifetime broadenings shape the peaks in the RIXS plane. If fully resolved, their spectral profiles are symmetric in the incident energy and energy transfer direction. Resonant 1s excitations appear along a diagonal line in the $(\Omega, \Omega-\omega)$ RIXS plane at constant emission energy $\omega$. The theoretical model system in Figure 3 shows two $1 \mathrm{~s}$ resonances on the diagonal line at 6542 and $6544 \mathrm{eV}$ incident energy. A third peak occurs in the vertical or final-state energy direction at the same incident energy as the first 1 s resonance. The $2 p$ final-state interactions give rise to additional peaks for a $1 \mathrm{~s}$ resonance at the same incident energy shifted toward higher energy transfer. In the energy scheme of Figure 1, these additional final states are indicated by the dotted line, and in Figure 3 they are indicated by the off-diagonal peak. This is an important observation for the interpretation of the experimental spectra.
Figure 3 shows three line plots extracted from the RIXS plane. Two sets of line plots were obtained by integrating the spectral intensity along the incident energy and the energy transfer, respectively, within certain narrow limits around the resonances. We use the acronym CET (constant energy transfer) to denote those line plots where the intensity is integrated over the energy transfer $\Omega-\omega$ and plotted versus the incident energy $\Omega$. The resulting spectrum corresponds to a constant final-state scan over a certain range of final states. The lifetime broadening in such a scan arises from the $1 \mathrm{~s}$ core hole $\left(\Gamma_{\mathrm{K}}\right)$. Note that such a scan becomes identical to a regular K-edge XANES scan if the integrated range extends over all final-state energies. If we include in a CET line plot all final states with $2 \mathrm{p}_{3 / 2} 3 \mathrm{~d}^{n+1}\left(\mathrm{~L}_{3}\right)$ configurations, we obtain a good approximation to a $1 \mathrm{~s}$ to $3 \mathrm{~d}$ absorption spectrum. ${ }^{35}$ This will be our approach when interpreting the experimental data along the incident energy axis.

We use the acronym CIE (constant incident energy) to denote those line plots where the intensity is integrated over the incident energy $\Omega$ and plotted versus the energy transfer $\Omega-\omega$. The resulting spectrum corresponds to a constant intermediate-state scan over a certain range of intermediate states. A CIE line plot in $1 s 2 p$ RIXS is formed by the same final-state configuration as an L-edge absorption spectrum. The line broadening arises solely from the final-state lifetime $\Gamma_{\mathrm{L}}$.

A diagonal cut through the $(\Omega, \Omega-\omega)$ RIXS plane corresponds to a scan at constant emission energy $\omega$. We denote these lines as CEE (constant emission energy) scans. "K $\alpha_{1}$ detected CEE scans" are then scans where the emission energy $\omega$ is set to the $\mathrm{Mn} \mathrm{K} \alpha_{1}$ fluorescence energy. Figure 3 illustrates that the peaks in a CEE scan appear sharper than $\Gamma_{K}$ and $\Gamma_{L}$ because the scan is performed in the RIXS plane at $45^{\circ}$ with respect to the lifetime broadenings. ${ }^{52-54}$ The CEE spectral shape can be calculated from the Kramers-Heisenberg equation. We note that a CEE plot yields a good approximation to an absorption spectrum if no final-state interactions and multielectron excitations are present.

\section{Materials and Methods}

Mn Model Complexes. $\mathrm{MnO}, \mathrm{Mn}_{3} \mathrm{O}_{4}, \mathrm{Mn}_{2} \mathrm{O}_{3} \mathrm{MnO}_{2}, \mathrm{Mn}^{\mathrm{II}}(\mathrm{acac})_{2^{-}}$ $\left(\mathrm{H}_{2} \mathrm{O}\right)_{2}$, and $\mathrm{Mn}^{\mathrm{III}}(\mathrm{acac})_{3}$ were purchased from Alpha products and used as is. $\mathrm{Mn}^{\mathrm{IV}}$ (salicylate $)_{2}$ (bipy) and $\left[\mathrm{Mn}{ }^{\mathrm{III}}(5-\mathrm{Cl}-\mathrm{Salpn})\left(\mathrm{CH}_{3} \mathrm{OH}\right)_{2}\right]\left(\mathrm{O}_{3} \mathrm{SCF}_{3}\right)$ were prepared by literature methods. ${ }^{55,56}$ To minimize fluorescence "saturation" and transmission "leakage" artifacts, the samples were made optically thin by diluting the pure sample with boron nitride..$^{57}$

PS II Preparation. PS II membranes were prepared from fresh spinach leaves by a 2-min incubation of the isolated thylakoids with the detergent Triton X-100 (Sigma) and subsequent centrifugation. ${ }^{58,59}$ These native PS II samples were suspended in the $30 \%$ glycerol buffer (pH 6.5, $50 \mathrm{mM}$ MES, $15 \mathrm{mM} \mathrm{NaCl}, 5 \mathrm{mM} \mathrm{MgCl}_{2}, 5 \mathrm{mM} \mathrm{CaCl}_{2}$ ) and centrifuged at $17500 \mathrm{rpm}$ for an hour. The resulting pellets were transferred to the Lucite sample holders (16 holders, $80 \mu \mathrm{L}$ each, 35 $\mathrm{mg}$ of $\mathrm{Chl} / \mathrm{mL}$ ) designed to fit into both EPR and X-ray cryostats. After

(52) Hämäläinen, K.; Siddons, D. P.; Hastings, J. B.; Berman, L. E. Phys. Rev. Lett. 1991, 67, 2850-2853.

(53) de Groot, F. M. F.; Krisch, M. H.; Vogel, J. Phys. Rev. B 2002, 66, 195112

(54) Hayashi, H.; Udagawa, Y.; Caliebe, W.; Kao, C.-C. Chem. Phys. Lett. 2003 , $371,125-130$

(55) Pavacik, P. S.; Huffman, J. C.; Christou, G. J. Chem. Soc., Chem. Comm. 1986, $43-44$

(56) Larson, E. J.; Pecoraro, V. L. J. Am. Chem. Soc. 1991, 113, 3810-3818

(57) Goulon, J.; Goulon-Ginet, C.; Cortes, R.; Dubois, J. M. J. Phys. (Paris) 1982, 43, 539-548

(58) Berthold, D. A.; Babcock, G. T.; Yocum, C. F. FEBS Lett. 1981, 134 $231-234$

(59) Kuwabara, T.; Murata, N. Plant Cell Physiol. 1982, 23, 533-539. 
the dark-adaptation for $1 \mathrm{~h}$ at room temperature, the samples were predominantly in the $S_{1}$ state. These samples were stored in liquid nitrogen until used. Half of the $S_{1}$ sample holders were taken out later and illuminated with a $400 \mathrm{~W}$ tungsten lamp at $200 \mathrm{~K}$ for $10 \mathrm{~min}$ to establish the $S_{2}$ state. ${ }^{60}$ The $S_{2}$ state samples were then stored in liquid nitrogen.

All samples were characterized by EPR spectroscopy at $8 \mathrm{~K}$ to ensure the quality of the samples before and after illumination. Spectra were collected using a Varian E-109 EPR spectrometer attached with an Air Products Helitran liquid helium cryostat. The generation of the $S_{2}$ state after the illumination was checked by the intensity of EPR multiline signal characteristic to the $S_{2}$ state.

Oxygen activity for the PS II sample was assayed under saturating white light using a Clark-type oxygen electrode (Yellow Springs Instruments) as described in the literature. ${ }^{61}$ The enzyme activity in the present study ranged from 350 to $380 \mu \mathrm{mol} \mathrm{O}_{2} / \mathrm{mg}$ chlorophyll (Chl)/ h.

Synchrotron Beamline. The RIXS data were recorded on the Bio CAT undulator beamline 18-ID at the Advanced Photon Source. ${ }^{62}$ The energy of the incoming synchrotron beam was selected by means of a liquid nitrogen-cooled Si double-crystal monochromator with a $(1,1,1)$ orientation. The sample was oriented at $45^{\circ}$ relative to the incident beam, and the beam size on the sample was $2.8 \mathrm{~mm}$ horizontal and 0.2 $\mathrm{mm}$ vertical fwhm. Higher harmonics were rejected by the focusing mirror. Using the fundamental undulator peak, the maximum incident flux was $10^{13}$ photons $/ \mathrm{s}$ at $6.5 \mathrm{keV}$. For some samples, the incident beam intensity was attenuated by $\mathrm{Al}$ foils to prevent detector saturation and/or sample radiation damage. The incident X-ray monochromator energy bandwidth was approximately $1.0 \mathrm{eV}$ at $6540 \mathrm{eV}$. The BioCAT beamline operated in continuous monochromator scan mode. Data were acquired "on-the-fly" during motor motion, minimizing the dead time per scan. To reduce sample illumination, a beam shutter was automatically inserted when no data were acquired during motor motions (monochromator or sample positioning). The monochromator energy calibration was simultaneously checked by recording the $\mathrm{K}$ pre-edge peak of $\mathrm{KMnO}_{4}$ using two additional ion chambers downstream of the sample.

Spectroscopy Measurements. The scattered X-rays were collected by means of a crystal array spectrometer. ${ }^{38}$ The $(3,3,3)$ Bragg reflection of four spherically bent Ge crystals arranged in a Rowland geometry was used. The analyzer crystals captured a solid angle of $3.4 \times 10^{-2}$ sr. A liquid nitrogen-cooled solid state $(\mathrm{Ge})$ detector was placed at the common focus of the four crystals, and the entire emitted beam path was enclosed by a He-filled bag. The narrow energy bandwidth of the Ge detector $(\sim 200 \mathrm{eV}$ at $5.9 \mathrm{keV})$ was used to window out unwanted $\mathrm{X}$-ray events and thus to improve the signal-to-background ratio. We determined an intrinsic analyzer bandwidth of $0.8 \mathrm{eV}$ by measuring the elastically scattered peak and assuming a $1.0 \mathrm{eV}$ primary monochromator energy bandwidth. Energy calibration of the spectrometer was achieved by measuring the absolute angle of the Bragg reflection using optical tools. We estimated that the upper limit for the error of the absolute energy calibration was $1 \mathrm{eV}$.

In the geometry the spectrometer was used, the focusing or Rowland circles were oriented perpendicular to the synchrotron plane. The vertical direction was therefore the energy-dispersive direction. Vertical movements of the synchrotron beam thus resulted in a change of the energy calibration of the spectrometer. By using a beam position monitor, we were able to correct the spectrometer energy calibration

(60) Brudvig, G. W.; Casey, J. L.; Sauer, K. Biochim. Biophys. Acta 1982, 723, $366-371$

(61) DeRose, V. J.; Mukerji, I.; Latimer, M. J.; Yachandra, V. K.; Sauer, K.; Klein, M. P. J. Am. Chem. Soc. 1994, 116, 5239-5249.

(62) Bunker, G. B.; Irving, T.; Black, E.; Zhang, K.; Fischetti, R.; Wang, S.; Stepanov, S. BioCAT Undulator Beamline at APS. In Synchrotron Radiation Instrumentation, 10th U.S. National Conference, Ithaca, NY, June, 1997; Fontes, E., Ed.; American Institute of Physics: Woodbury, NY, 1997; p 16. for vertical beam movements and thus minimize the experimental error in the direction of the energy transfer. The precision of the beam position monitor was better than $50 \mu \mathrm{m}$. We can thus give an upper limit for the absolute error of the energy transfer of $80 \mathrm{meV}$. Scans of the scattered X-rays were taken between 5875.6 and $5914.1 \mathrm{eV}$, corresponding to Bragg angles of $75.7^{\circ}$ and $74.3^{\circ}$, respectively. The horizontal angle between the incident synchrotron beam direction and the solid angle covered by the four analyzer crystals ranged from $73.9^{\circ}$ to $106.1^{\circ}$. Energy scans of the scattered X-rays were achieved by moving the analyzer crystals vertically. ${ }^{38}$ For the model compounds (PS II samples) the scan step size was $0.05(0.12) \mathrm{mm}$, which translates to energy steps of $0.08(0.21) \mathrm{eV}$ at $5875.6 \mathrm{eV}$ and $0.1(0.23) \mathrm{eV}$ at $5914.1 \mathrm{eV}$ on the energy transfer axis. The count rates in the continuous monochromator scans were integrated over steps of $0.1 \mathrm{eV}$.

All samples were kept in a liquid He-cooled cryostat below $10 \mathrm{~K}$ and surrounded by an exchange gas ( $\mathrm{He}$ ) at ambient pressure. Radiation damage studies were thoroughly performed for the PS II samples as well as for the model compounds by recording $\mathrm{K} \alpha_{1}$-detected CEE scans. The beam position on the sample could be adjusted vertically and horizontally, and the total illumination time per sample position was kept below the time limit when damage occurred. To obtain the twodimensional RIXS plane, we recorded CEE scans with the fluorescence energy changed stepwise. We took a total of 401 (134) CEE scans for the model compounds (PS II samples) to build up the experimental RIXS planes. Each scan corresponds to a diagonal cut in the $(\Omega, \Omega-$ $\omega)$ plane. The beam position on the sample was changed with every scan, and the maximum scan time was $30 \mathrm{~s}$ for the model compounds, depending on the time limit when radiation damage occurred. The incident beam intensity had to be attenuated to $60 \%$, and the total illumination time was minimized to 2 seconds for the PS II samples to avoid radiation damage. To correct for $\mathrm{Mn}$ concentration variations across the sample, we measured the scattered X-ray intensity at a constant incident energy chosen at the pre-edge absorption maximum for each sample position. The intensity of the signal varied in a typical sample by less than $15 \%$.

For both states, $S_{1}$ and $S_{2}$, the spectra of four PS II samples, each with $80 \mu \mathrm{L}$ protein solution, were added up. The total data acquisition times were about $4 \mathrm{~h}(2 \mathrm{~s} / \mathrm{spot})$ for each S-state and 3-5.5 h (max. 30 $\mathrm{s} / \mathrm{spot}$ ) for each of the model compounds, including all dead times and concentration correction scans. The total counts in the peak maximum of the RIXS plane are about 120 counts for the PS II samples on a background of about four counts. The integrated CET and CIE line plots have total counts in the peak maximum between 2500 and 3500.

Data Analysis. Each CEE scan was corrected for variations in the incident intensity $I_{0}$, and all CEE scans that form an RIXS plane were corrected relative to each other with respect to $I_{0}$ and Mn concentration variations across the sample. The experimental RIXS plane was splined on an energy grid with equal energy steps of $0.1 \mathrm{eV}$ in both directions ( $0.2 \mathrm{eV}$ for PS II). A constant background was subtracted from all RIXS spectra. A running average including five data points in the energy transfer as well as the monochromator direction was applied in the case of the PS II samples.

Intensity due to excitations at incident energies higher than the preedge was subtracted from the RIXS spectra similar to main edge subtraction in conventional absorption spectroscopy. In the procedure chosen here, scans parallel to the incident energy axis at a constant energy transfer (CET line plots) were fitted to PearsonVII functions. The PearsonVII function simulates a Voigt profile, which in turn is a convolution of a Gaussian and a Lorentzian line profile. Details for the fitting procedure are given in the Supporting Information. Several fits with varying starting parameters and fit constraints were performed for every experimental RIXS plane shown here. An error was determined for the moment analysis from the different fit results (vide infra). We note that the pre-edge structure in the analysis of conventional absorption spectra is usually separated by fitting a cubic spline function or an arctangent function to the main edge intensity. Both approaches 
are considerable simplifications of the real main edge shape. The arctangent functional shape only describes $1 \mathrm{~s}$ to $\epsilon \mathrm{p}$ continuum excitations, neglecting all resonance effects, ${ }^{63}$ and a cubic spline fit strongly depends on the included data range. RIXS spectroscopy considerably improves the pre-edge extraction because the energy transfer direction is added, which separates the pre-edge from the main edge. Our fitting procedure, moreover, takes resonant excitations in the main edge into account. However, some uncertainty also remains here.

Difference spectra were created to display the spectral changes. Before subtraction, the spectra were normalized relative to each other to have equal integrated spectral areas of the pre-edge structure in the RIXS plane. In conventional absorption spectroscopy, the common procedure is to normalize the $\mathrm{K}$ main edge jump. Normalization to the pre-edge spectral area eliminates variations in the intensity of the preedge relative to the $\mathrm{K}$ main edge. Such variations can be due to, for example, stronger dipole admixture because of a change in the local symmetry at the Mn site. This is discussed by Westre et al. for ferric and ferrous compounds with $\mathrm{Fe}$ in $O_{h}$ and $T_{d}$ symmetry. ${ }^{19}$ In this study, we focused on energy splittings and relative shifts. We chose the preedge area normalization because this approach proved to be best-suited to display the spectral changes following Mn oxidation as we will show. We address the influence of dipole transitions in the Discussion section.

A first moment analysis was performed to quantify the information contained in the RIXS spectra:

$$
\mathrm{M}_{1}^{p}=\frac{\sum_{j} E_{j} \cdot I_{j}}{\sum_{j} I_{j}}
$$

where $E_{j}$ and $I_{j}$ are the energies and fluorescence intensities, respectively, of the jth data point. All data points with intensities greater than $p$ percent of the maximum intensity are included in the calculation of $\mathrm{M}_{1}{ }^{p}$. The first moment is a way to define the center-of-gravity energy of a spectral feature. We note that the statistical error of the first moment is insignificant even for the PS II spectra with relatively low total counts.

\section{Results}

Mn Oxides. The 1s2p RIXS spectra of the four Mn oxides are shown in Figure 4, with the contour plots showing the experimental spectra before and after subtraction of the main edge. The diagonal continuous streak of intensity at high incident energy and energy transfer is the rising Mn K main edge. The pre-edge merges into the main edge for the Mn oxides with oxidation states higher than II. Some uncertainty in the main edge subtraction is present at the transition point between preedge and main edge for those Mn compounds. This uncertainty is expressed as errors in the moment analysis (vide infra).

The spectrum for $\mathrm{MnO}$ in particular nicely shows how the RIXS plane enables separation of the pre-edge from the main edge. The $\mathrm{MnO}$ pre-edge structure stretches diagonally over about $2 \mathrm{eV}$, indicating that at least two strong resonances form the spectrum. $\mathrm{MnO}, \mathrm{Mn}_{3} \mathrm{O}_{4}$, and $\mathrm{Mn}_{2} \mathrm{O}_{3}$ show pre-edge intensity at around $6540.5 \mathrm{eV}$. However, for $\mathrm{Mn}_{3} \mathrm{O}_{4}$ and $\mathrm{Mn}_{2} \mathrm{O}_{3}$ the peak appears much sharper and symmetric compared to that of $\mathrm{MnO}$. This spectral feature therefore has a different origin in $\mathrm{Mn}_{3} \mathrm{O}_{4}$ and $\mathrm{Mn}_{2} \mathrm{O}_{3}$ than in $\mathrm{MnO}$. Multiplet calculations can explain the different origins as discussed below. It is an important observation in context with the PS II data that spectral intensity at $6540.5 \mathrm{eV}$ does not necessarily indicate a $\mathrm{Mn}(\mathrm{II})$ species in the sample.

(63) Breinig, M.; Chen, M. H.; Ice, G. E.; Parente, F.; Crasemann, B.; Brown, G. S. Phys. Rev. A 1980, 22, 520-528.
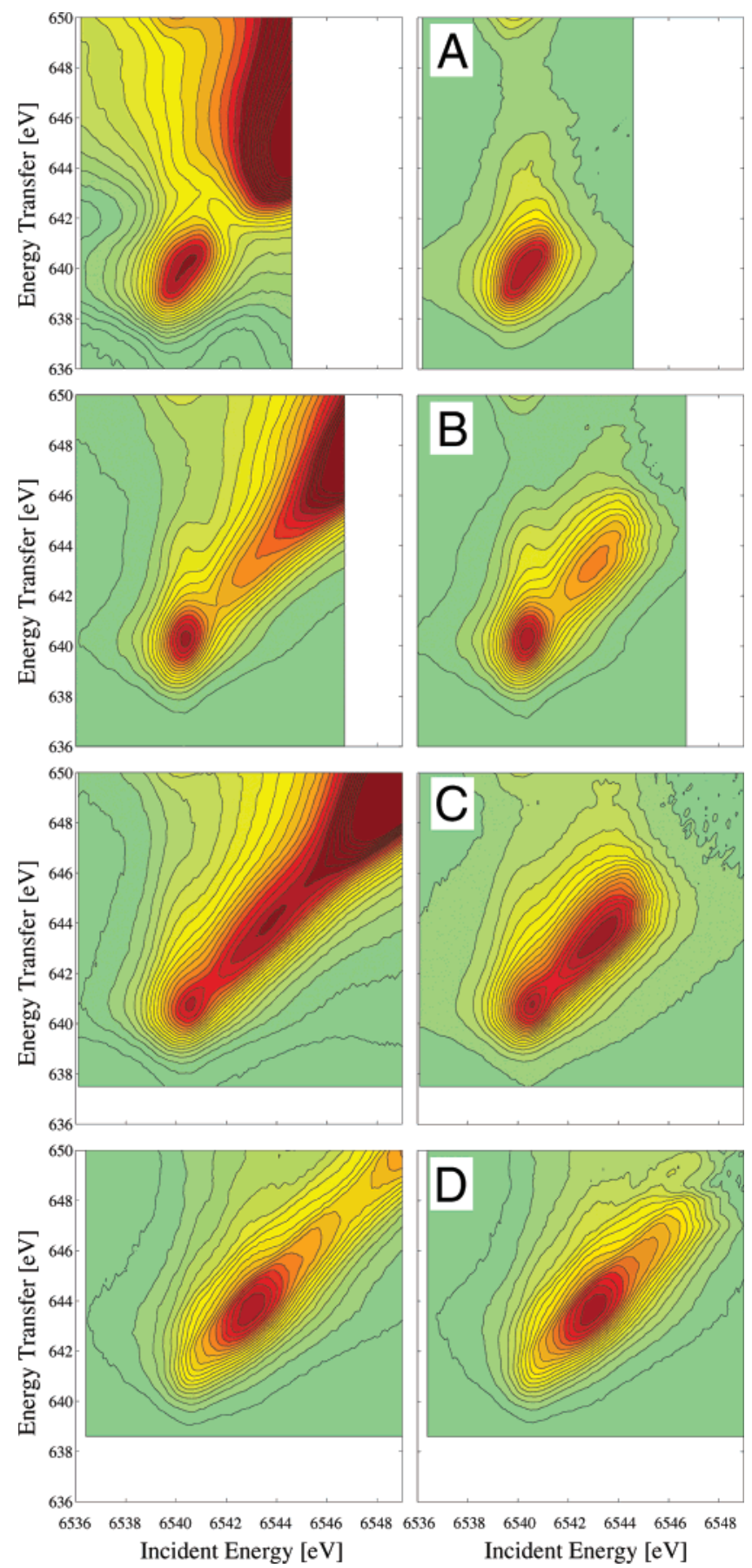

Figure 4. Contour plots of the $1 \mathrm{~s} 2 \mathrm{p}_{3 / 2}$ RIXS planes for Mn oxides: $\mathrm{MnO}$ (A), $\mathrm{Mn}_{3} \mathrm{O}_{4}$ (B), $\mathrm{Mn}_{2} \mathrm{O}_{3}$ (C), and $\mathrm{MnO}_{2}$ (D). The plots on the right show the $\mathrm{K}$ pre-edge features after subtraction of the main edge. The energy axes are identical for all plots.

For $\mathrm{Mn}_{3} \mathrm{O}_{4}$ and $\mathrm{Mn}_{2} \mathrm{O}_{3}$, there is additional spectral intensity around $6543.5 \mathrm{eV}$ incident energy. Both spectra show a similar overall shape with a sharp resonance at low energies and a broad structure at higher energies. The spectrum is different for $\mathrm{MnO}_{2}$ where only one broad structure is visible with one maximum and pronounced tails toward higher and lower energies.

All peaks in the experimental spectra show asymmetric line shapes with shoulders in the direction of the energy transfer. The asymmetries arise from off-diagonal features as discussed in context with the theoretical model system in Figure 3. 

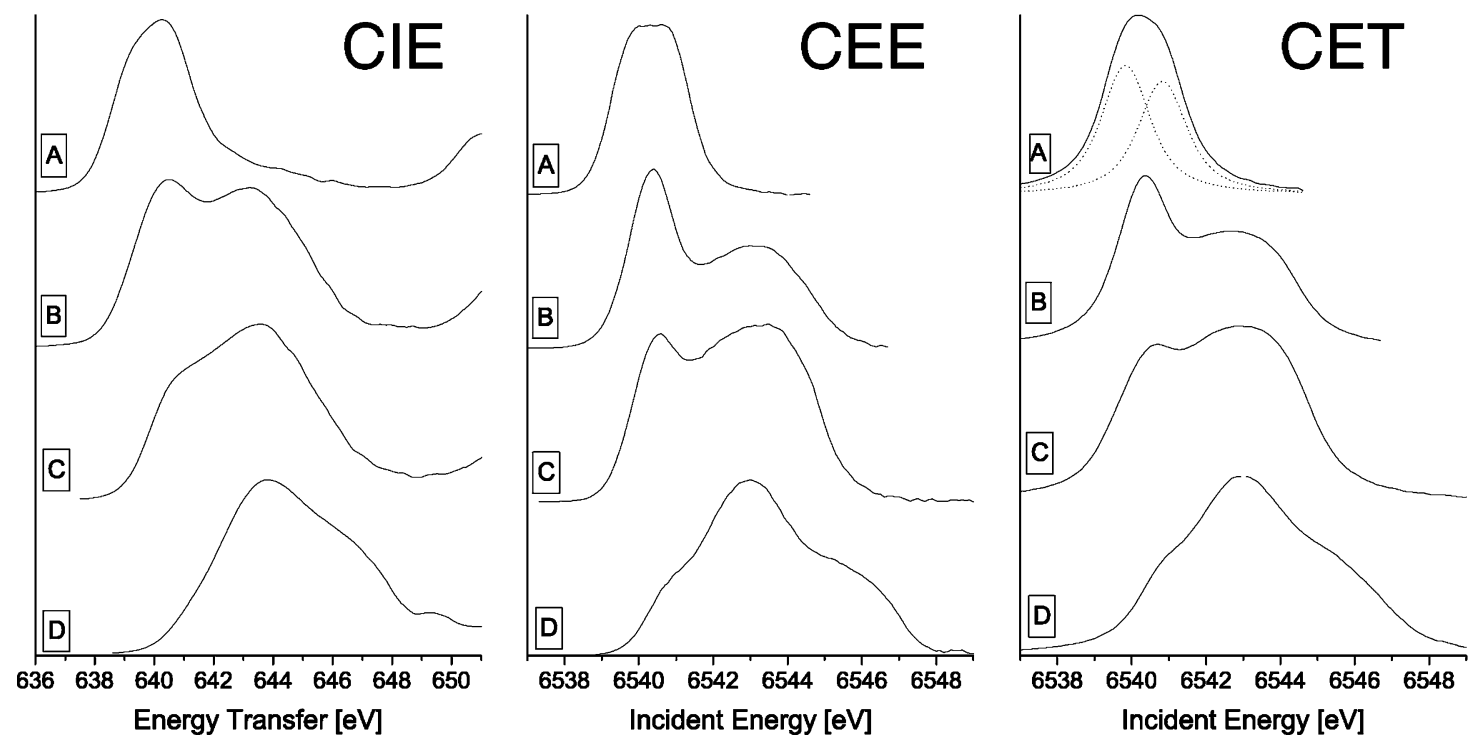

Figure 5. Line plots extracted from the RIXS planes for the four Mn oxides. Left panel: RIXS intensity integrated over the incident energy plotted versus the energy transfer (CIE). Center panel: Diagonal cuts through RIXS planes at constant emission energy (CEE). Right panel: RIXS intensity integrated over the energy transfer plotted versus the incident energy (CET). The MnO (A) CET line plot was fitted using two Voigt profiles (dotted curves).

Table 1. First Moment Analysis $\mathrm{M}_{1} 20 \%$ for the Pre-Edge Structure of the Mn Oxides

\begin{tabular}{llcccc}
\hline & $\begin{array}{c}\text { formal } \\
\text { oxidation } \\
\text { state }\end{array}$ & $\begin{array}{c}\text { incident } \\
\text { energy } \\
{[+6500 \mathrm{eV}]}\end{array}$ & $\begin{array}{c}\Delta E \\
{[\mathrm{eV}]}\end{array}$ & $\begin{array}{c}\text { energy } \\
\text { transfer } \\
{[+600 \mathrm{eV}]}\end{array}$ & $\begin{array}{c}\Delta E \\
{[\mathrm{eV}]}\end{array}$ \\
\hline (A) $\mathrm{MnO}$ & II & $40.3 \pm 0.1$ & & $40.2 \pm 0.1$ & \\
& & & $1.4 \pm 0.2$ & & $1.9 \pm 0.2$ \\
(B) $\mathrm{Mn}_{3} \mathrm{O}_{4}$ & (II,III,III) & $41.7 \pm 0.1$ & & $42.1 \pm 0.1$ & $0.9 \pm 0.2$ \\
(C) $\mathrm{Mn}_{2} \mathrm{O}_{3}$ & III & $42.3 \pm 0.1$ & $0.6 \pm 0.2$ & $43.0 \pm 0.1$ & \\
(D) $\mathrm{MnO}_{2}$ & IV & $43.4 \pm 0.1$ & & $44.4 \pm 0.1$ & \\
& & & & &
\end{tabular}

However, unlike in the theoretical plot, these additional features are not resolved from the strong pre-edge peaks in the experimental spectra. They are caused by electron-electron interactions that occur in the $2 \mathrm{p}^{5} 3 \mathrm{~d}^{n+1}$ final states and not in the $1 \mathrm{~s} 3 \mathrm{~d}^{n+1}$ intermediate states.

We performed a first moment analysis (cf. eq 2, $p=20 \%$ ) for all experimental spectra after edge subtraction. The results are shown in Table 1 . The values for $\mathrm{M}_{1}{ }^{20 \%}$ increase with the average Mn oxidation state in both directions of the RIXS plane, but the chemical shifts are larger in the energy transfer than in the incident energy direction. We attribute the more pronounced chemical sensitivity in the energy transfer direction to the strong (2p,3d) electron-electron final-state interactions. Between $\mathrm{Mn}(\mathrm{II})$ in $\mathrm{MnO}$ and $\mathrm{Mn}$ (III) in $\mathrm{Mn}_{2} \mathrm{O}_{3}$ we observe a shift of the incident energy first moment of $\Delta E=2.0 \pm 0.2 \mathrm{eV}$. For the mixed-valent $\mathrm{Mn}_{3} \mathrm{O}_{4}$ we would expect a shift of $2.0 \mathrm{eV} * 2 / 3$ $=1.33 \mathrm{eV}$ relative to $\mathrm{MnO}$. This is consistent with the observed value of $1.4 \pm 0.2 \mathrm{eV}$. The first moment shift in the Mn oxides thus appears to follow the Mn oxidation state and is in this case independent of the local geometry.

Figure 5 shows the three types of line plots for the Mn oxides that we introduced earlier. The entire incident energy range shown in the contour plots in Figure 4 was used to produce the CIE plots. For the CET plots the range was from 637 to 648 $\mathrm{eV}$ on the energy transfer axis for all samples. CEE plots were generated from diagonal cuts through the RIXS plane in the maximum of the pre-edge structure. Comparison of the CEE with the CET line plots shows the line-sharpening effect in the CEE scans as explained earlier. It is most evident for $\mathrm{Mn}_{3} \mathrm{O}_{4}$ (B) and $\mathrm{Mn}_{2} \mathrm{O}_{3}(\mathrm{C})$. The relative intensities of the peaks in a CEE plot strongly depend on where the diagonal cut through the RIXS plane is generated; this is because of the $(2 p, 3 d)$ finalstate multiplet interaction. Different line shapes between CEE and CET scans can thus be observed.

The CEE and CET plots for MnO clearly show that the preedge structure arises from at least two resonances. A fit of the CET plot (this plot represents the absorption cross section) using two Voigt line profiles with identical fwhm yields a splitting of the two resonances of $1.1 \mathrm{eV}$. This value gives the crystal field splitting $10 \mathrm{Dq}$ between the $\mathrm{t}_{2 \mathrm{~g}}$ and the $\mathrm{e}_{\mathrm{g}}$ orbitals in the presence of a Mn 1s hole. We note that a core hole excited state yields a $10 \mathrm{Dq}$ value that is only about $80 \%$ of those obtained from optical data. ${ }^{48,64}$ The direct observation of $10 \mathrm{Dq}$ is only possible because of the absence of strong (3d,3d) multiplet splitting. The same argument applies to ferric $O_{h}$ complexes as discussed by Westre et al. ${ }^{19}$ The $(3 \mathrm{~d}, 3 \mathrm{~d})$ interaction dominates the pre-edge spectral shapes of all Mn samples with oxidation states higher than II giving rise to numerous final states (vide infra). A fit of the corresponding line plots bears large ambiguity and does not give new insights.

The CIE line plot for $\mathrm{MnO}$ shows weak structures on the high energy transfer side $(\sim 644 \mathrm{eV})$ of the main peak. These structures are also visible in the conventional L-edge absorption spectra and were found to be indicative of a high-spin configuration. $^{28,48,49}$ They are caused by the $(2 \mathrm{p}, 3 \mathrm{~d})$ multiplet interactions and are therefore absent in the CET line plot. This demonstrates the similarities between L-edge and 1s2p RIXS spectroscopy and shows that $1 \mathrm{~s} 2 \mathrm{p}$ RIXS is sensitive to the metal ion spin state.

Mn Coordination Complexes and PS II. The 1s $2 p$ RIXS spectra of the four coordination complexes as well as the $S_{1}$

(64) Lever, A. B. P. Inorganic Electronic Spectroscopy; Elsevier: Amsterdam, 1984. 



Figure 6. Contour plots of the $1 \mathrm{~s} 2 \mathrm{p}_{3 / 2}$ RIXS planes for the four molecular complexes $\mathrm{Mn}^{\mathrm{II}}(\mathrm{acac})_{2}\left(\mathrm{H}_{2} \mathrm{O}\right)_{2}(\mathrm{E}), \mathrm{Mn}^{\mathrm{III}}(\mathrm{acac})_{3}(\mathrm{~F}),\left[\mathrm{Mn}^{\mathrm{III}}(5-\mathrm{Cl}-\mathrm{Salpn})\left(\mathrm{CH}_{3}\right.\right.$ $\left.\mathrm{OH})_{2}\right]\left(\mathrm{O}_{3} \mathrm{SCF}_{3}\right)(\mathrm{G})$, and $\mathrm{Mn}^{\mathrm{IV}}(\mathrm{sal})_{2}$ (bipy) $(\mathrm{H})$.

and $S_{2}$ states of PS II are shown in Figures 6 and 7, respectively. The corresponding line plots are given in Figure 8, and the first moments are listed in Table 2. A striking similarity in the spectral shapes is found between the $\mathrm{Mn}(\mathrm{II}), \mathrm{Mn}(\mathrm{III})$, and $\mathrm{Mn}(\mathrm{IV})$ coordination complexes and $\mathrm{MnO}, \mathrm{Mn}_{3} \mathrm{O}_{4}$, and $\mathrm{Mn}_{2} \mathrm{O}_{3}$. The $\mathrm{Mn}(\mathrm{II})$ complex shows one pre-edge structure such as $\mathrm{MnO}$. The structure, however, is less broad, indicating a smaller crystal field splitting in the coordination complex. Indeed, by fitting the CET line plot we obtain a value of 0.7 $\mathrm{eV}$ for $10 \mathrm{Dq}$ in the $1 \mathrm{~s}$ excited state. The feature on the high energy transfer side in the CIE plot appears more pronounced for the $\mathrm{Mn}$ (II) coordination complex than that for MnO. This
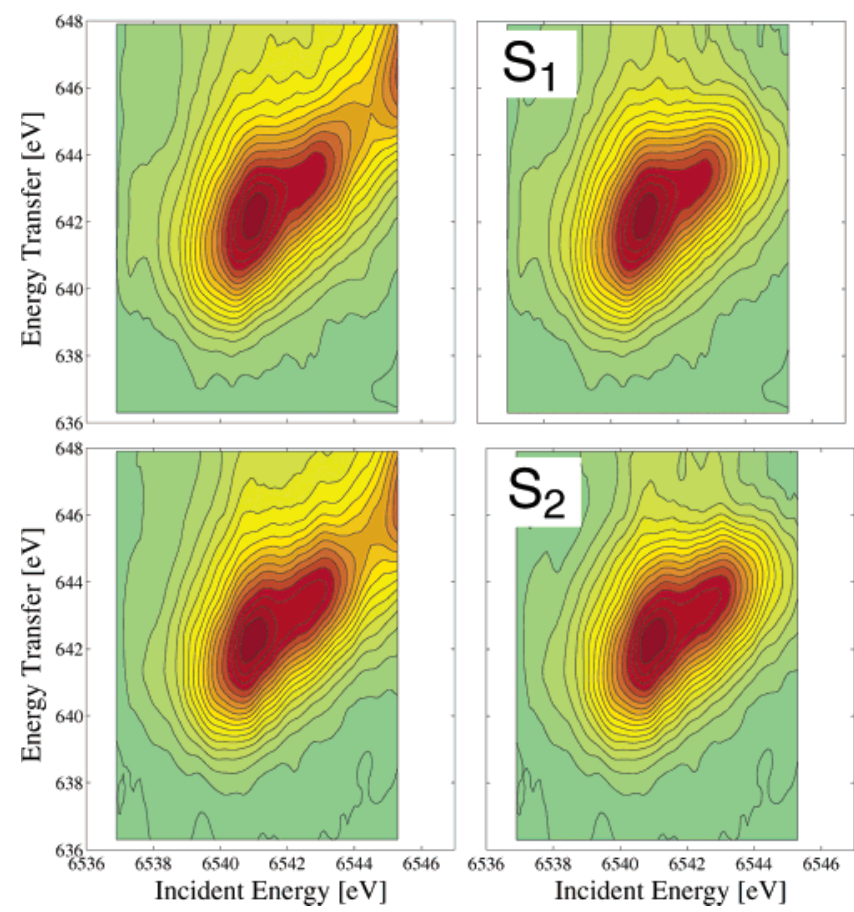

Figure 7. Contour plots of the $1 \mathrm{~s} 2 \mathrm{p}_{3 / 2}$ RIXS planes for PS II in the $\mathrm{S}_{1}$ and $\mathrm{S}_{2}$ states.

suggests a stronger $(2 \mathrm{p}, 3 \mathrm{~d})$ final-state interaction in the case of the molecular complex. It is noteworthy that we obtain identical first moments for $\mathrm{MnO}$ and $\mathrm{Mn}^{\mathrm{II}}(\mathrm{acac})_{2}\left(\mathrm{H}_{2} \mathrm{O}\right)_{2}$ in the incident energy direction but a shift of $0.3 \mathrm{eV}$ to lower energy transfer for the coordination complex compared to the oxide.

As with the Mn oxides, we observe a rise of intensity at higher incident energies with increasing oxidation state. Again we observe a rather sharp peak at low energies and a broad band at high energies. The Mn(IV) coordination complex displays a distinct spectral shape in the contour plot. Unlike all other Mn models with oxidation states higher than II, the two structures do not lie on a straight diagonal line but appear curved toward larger energy transfer. This results in considerably different spectral shapes for the CEE and CET line plots.

The first moments of the coordination complexes (cf. Table 2) show the same trends as the Mn oxides. The values increase with the formal oxidation state, and the increase is larger in the energy transfer direction. The two $\mathrm{Mn}$ (III) complexes do not have identical first moment positions but the values for $\left[\mathrm{Mn}^{\mathrm{III}}(5\right.$ $\left.\mathrm{Cl}-\mathrm{Salpn})\left(\mathrm{CH}_{3} \mathrm{OH}\right)_{2}\right]\left(\mathrm{O}_{3} \mathrm{SCF}_{3}\right)$ are shifted toward higher energies but stay below the values for the Mn(IV) complex.

For the PS II samples, the first peak appears broader than that for the model compounds, and the two structures are not as well-separated. The PS II spectra show an average of the four $\mathrm{Mn}$ atoms in the tetranuclear cluster that all have a more or less different electronic structure, and as a result, the spectral features become more diffuse. The overall spectral shape, however, is still similar to that of the Mn model systems. The incident energy $\mathrm{M}_{1}{ }^{20 \%}$ values for the PS II samples are lower than those for the Mn(IV) complex and larger than those for $\mathrm{Mn}^{\mathrm{III}}(\mathrm{acac})_{3}$, in agreement with the proposed oxidation state of $\mathrm{Mn}\left(\mathrm{III}_{2}, \mathrm{IV}_{2}\right)$ and $\mathrm{Mn}\left(\mathrm{III}, \mathrm{IV}_{3}\right)$ for $\mathrm{S}_{1}$ and $\mathrm{S}_{2}$, respectively. The value for $\left[\mathrm{Mn}^{\mathrm{III}}(5-\mathrm{Cl}-\mathrm{Salpn})\left(\mathrm{CH}_{3} \mathrm{OH}\right)_{2}\right]\left(\mathrm{O}_{3} \mathrm{SCF}_{3}\right)$ is equal to PS 

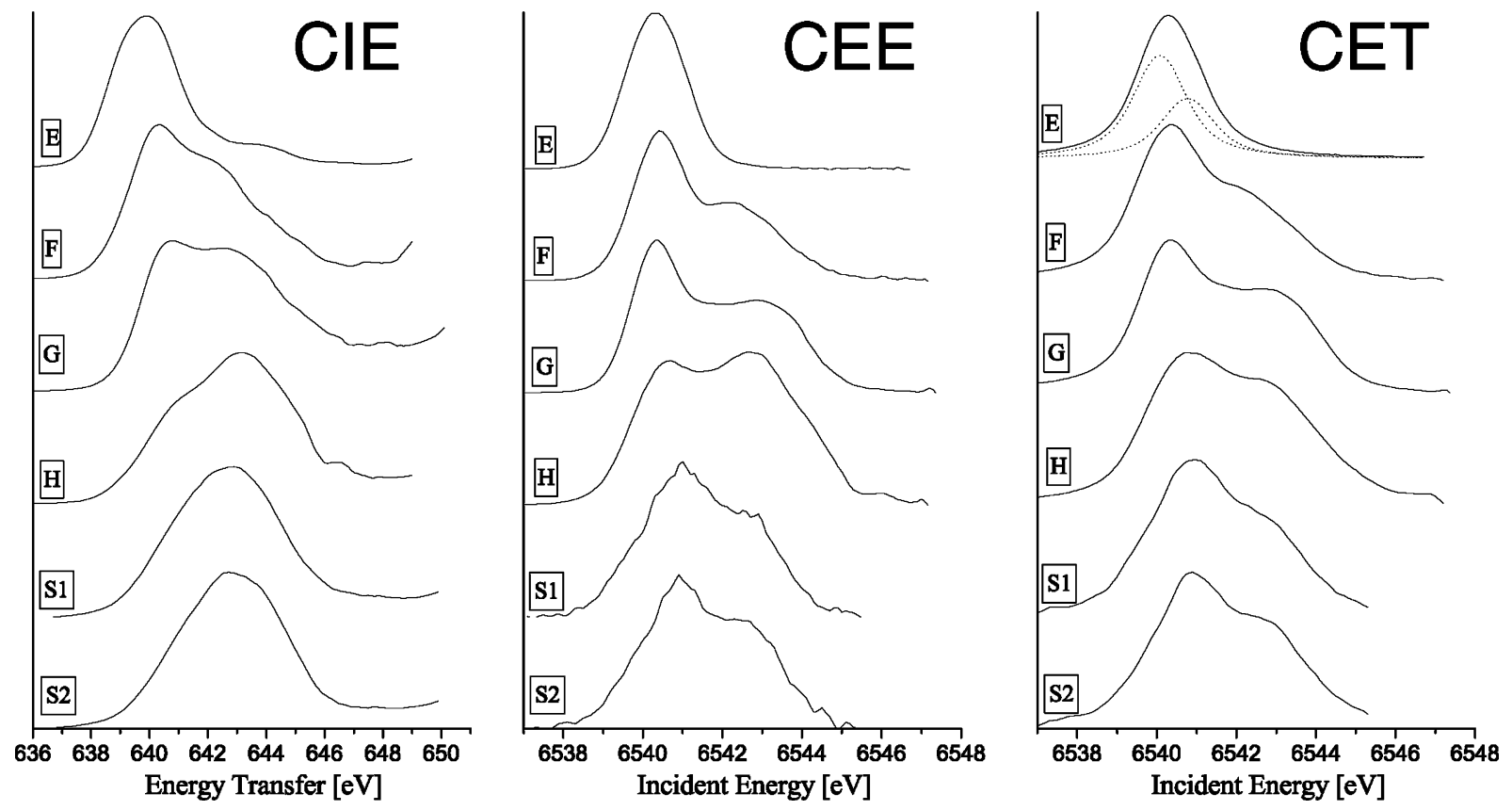

Figure 8. Line plots extracted from the RIXS planes for the four coordination complexes and the $\mathrm{S}_{1}$ and $\mathrm{S}_{2}$ states of PS II.

Table 2. First Moment Analysis $\mathrm{M}_{1} 20 \%$ for the Three Model Compounds and the $S_{1}$ and $S_{2}$ States of PS II

\begin{tabular}{|c|c|c|c|c|c|}
\hline & $\begin{array}{l}\text { formal } \\
\text { oxidation } \\
\text { state }\end{array}$ & $\begin{array}{c}\text { incident } \\
\text { energy } \\
{[+6500 \mathrm{eV}]}\end{array}$ & $\begin{array}{c}\Delta E \\
{[\mathrm{eV}]^{b}}\end{array}$ & $\begin{array}{c}\text { energy } \\
\text { transfer } \\
{[+600 \mathrm{eV}]}\end{array}$ & $\begin{array}{c}\Delta E \\
{[\mathrm{eV}]^{b}}\end{array}$ \\
\hline (E) $\mathrm{Mn}(\mathrm{acac})_{2}\left(\mathrm{H}_{2} \mathrm{O}\right)_{2}$ & II & $40.3 \pm 0.1$ & $0.8 \pm 0.2$ & $39.9 \pm 0.1$ & $1.3 \pm 0.2$ \\
\hline (F) $\mathrm{Mn}(\mathrm{acac})_{3}$ & III & $41.1 \pm 0.1$ & $0.7 \pm 0.2^{a}$ & $41.2 \pm 0.1$ & $1.5 \pm 0.2^{a}$ \\
\hline $\begin{array}{l}\text { (G) } \mathrm{Mn}^{\mathrm{IIII}}(5-\mathrm{Cl}-\mathrm{Salpn}) \\
\left(\mathrm{CH}_{3} \mathrm{OH}\right)_{2}\left(\mathrm{O}_{3} \mathrm{SCF}_{3}\right)\end{array}$ & III & $41.5 \pm 0.1$ & & $42.2 \pm 0.1$ & \\
\hline (H) $\mathrm{Mn}$ (sal) $)_{2}$ (bipy) & IV & $41.8 \pm 0.1$ & $0.3 \pm 0.2$ & $42.7 \pm 0.1$ & $0.5 \pm 0.2$ \\
\hline PS II $S_{1}$ & $\left(\mathrm{III}_{2}, \mathrm{IV}_{2}\right)$ & $41.5 \pm 0.2$ & $0.1 \pm 0.05^{b}$ & & $0.2 \pm 0.1$ \\
\hline PS II $S_{2}$ & $\left(\mathrm{III}, \mathrm{IV}_{3}\right)$ & $41.6 \pm 0.2$ & & $42.8 \pm 0.2$ & \\
\hline
\end{tabular}

${ }^{a}$ The shift relative to $\mathrm{Mn}(\mathrm{sal})_{2}$ (bipy) is given. ${ }^{b}$ The values for $\Delta E$ between $S_{1}$ and $S_{2}$ were obtained from different sets of fits, each with identical fit starting parameters as discussed in the text. Therefore, they show smaller errors.

II $\mathrm{S}_{1}$. The PS II results for $\mathrm{M}_{1}{ }^{20 \%}$ on the energy transfer axis match the $\mathrm{Mn}(\mathrm{IV})$ complex.

The absolute values for $\mathrm{M}_{1}{ }^{20 \%}$ for the two PS II samples are connected with a large error because of uncertainties in the fitting procedure for the background subtractions. We can give a smaller error for the relative shifts in $M_{1}{ }^{20 \%}$ between $S_{1}$ and $\mathrm{S}_{2}$. This was achieved by performing different background subtractions in the RIXS planes with equal fit starting parameters for the $S_{1}$ and $S_{2}$ spectra. For each set of starting parameters we thus obtain a fit for the $S_{1}$ and $S_{2}$ states. We then determined the shifts in $\mathrm{M}_{1}^{20 \%}$ for each set and found an average shift as given in Table 2. As in the model compounds, we find that the shift for the energy transfer moment is larger than that for the incident energy. The shifts between $S_{1}$ and $S_{2}$ are a factor of 7 to 8 smaller than those between $\mathrm{Mn}^{\mathrm{III}}(\mathrm{acac})_{3}$ and the $\mathrm{Mn}(\mathrm{IV})$ complex and about a factor of 3 smaller than those between $\left[\mathrm{Mn}^{\mathrm{III}}(5-\mathrm{Cl}-\mathrm{Salpn})\left(\mathrm{CH}_{3} \mathrm{OH}\right)_{2}\right]\left(\mathrm{O}_{3} \mathrm{SCF}_{3}\right)$ and the $\mathrm{Mn}(\mathrm{IV})$ complex.
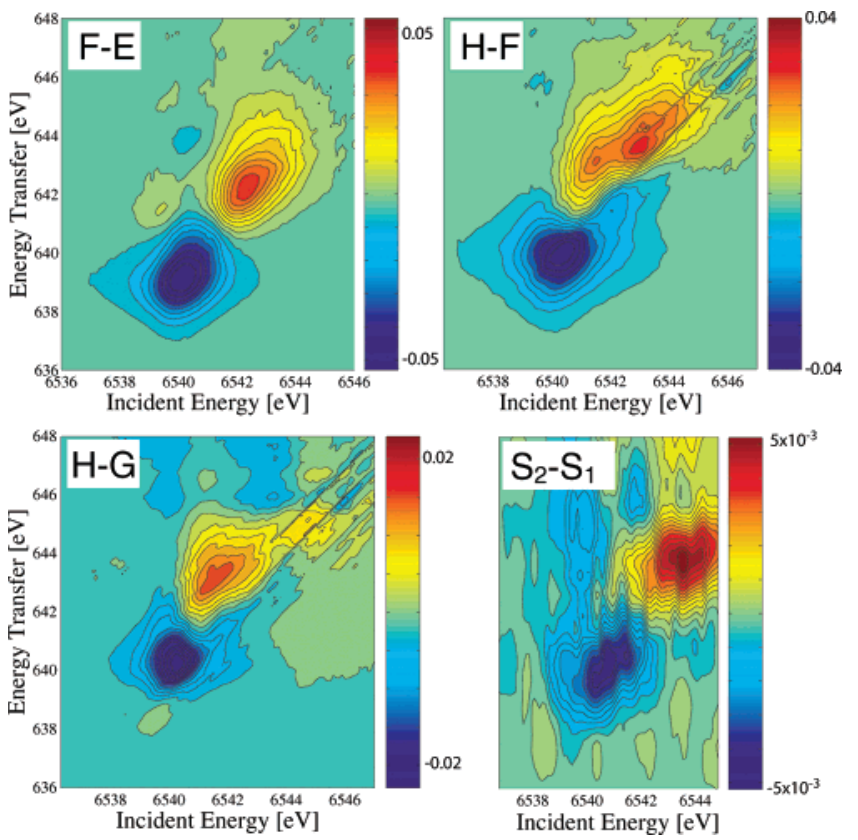

Figure 9. Difference contour plots of the $1 \mathrm{~s} 2 \mathrm{p}_{3 / 2}$ RIXS planes for three pairs of the four molecular model complexes as well as the $S_{1}$ and $S_{2}$ states of PSII. The intensities are shown in the color bars.

To display the spectral changes in the RIXS plane with increasing Mn oxidation state, we formed difference contours for three pairs of the coordination complexes $\left(\mathrm{Mn}^{\mathrm{III}}(\mathrm{acac})_{3}-\right.$ $\mathrm{Mn}^{\mathrm{II}}(\mathrm{acac})_{2}\left(\mathrm{H}_{2} \mathrm{O}\right)_{2}, \mathrm{Mn}^{\mathrm{IV}}(\text { sal })_{2}$ (bipy) $-\mathrm{Mn}^{\mathrm{III}}(\text { acac })_{3}$, and $\mathrm{Mn}^{\mathrm{IV}}$ (sal $)_{2}$ (bipy) - $\left.\left[\mathrm{Mn}^{\mathrm{III}}(5-\mathrm{Cl}-\mathrm{Salpn})\left(\mathrm{CH}_{3} \mathrm{OH}\right)_{2}\right]\left(\mathrm{O}_{3} \mathrm{SCF}_{3}\right)\right)$ as well as $\mathrm{S}_{2}-\mathrm{S}_{1}$ using the RIXS planes after edge subtraction (Figure $9)$. The spectral areas were normalized to one before subtraction, and the intensity gains and losses in the difference planes are therefore equal in magnitude; in other words, the integrated intensity in the difference contour plots is zero. Similar overall patterns for the strong changes are observed for all four 
difference spectra: Intensity is lost at low incident energy and energy transfer and gained at higher energies upon Mn oxidation.

A detailed analysis of the difference spectrum between the two S-states is limited because of insufficient statistics. The similarity in Figure 9 between the PS II and the model compounds difference contour plots, however, clearly shows that an oxidation of Mn occurs. We quantify the difference spectra by determining the areas with positive and negative values in the RIXS difference plane. We give the values in percentage of the pre-edge spectral area in the normalized RIXS plane and refer to them as "integrated intensity change". Since all RIXS spectral areas were normalized to one before subtraction, the percentage change is identical for the positive and the negative change and applies to both RIXS planes that were subtracted from each other. The values proved to be largely independent of the main edge subtraction procedure and therefore only show a small error. We obtain $31 \%$ and $30 \%$ for $\mathrm{Mn}^{\mathrm{III}}(\mathrm{acac})_{3}-\mathrm{Mn}^{\mathrm{II}}$ (acac $)_{2}\left(\mathrm{H}_{2} \mathrm{O}\right)_{2}$ and $\mathrm{Mn}^{\mathrm{IV}}(\mathrm{sal})_{2}$ (bipy) $-\mathrm{Mn}^{\mathrm{III}}(\text { acac })_{3}$, respectively. The difference spectrum $\mathrm{Mn}^{\mathrm{IV}}(\mathrm{sal})_{2}$ (bipy) $-\left[\mathrm{Mn}^{\mathrm{III}}(5-\mathrm{Cl}-\mathrm{Salpn})-\right.$ $\left.\left(\mathrm{CH}_{3} \mathrm{OH}\right)_{2}\right]\left(\mathrm{O}_{3} \mathrm{SCF}_{3}\right)$ shows a smaller change of $15 \%$. The error in both cases is $2 \%$. For $\mathrm{S}_{2}$ and $\mathrm{S}_{1}$ PS II, we obtain $4 \pm 1 \%$.

Both data reduction methods, first moment shifts or integrated intensity changes, give consistent results. We find that the model systems simulate the correct trends for PS II. When comparing the changes between $S_{1}$ and $S_{2}$ to the mononuclear model compounds, one has to multiply the PS II values by a factor of 4 because one oxidizing equivalent per four $\mathrm{Mn}$ ions is transferred in the $\mathrm{S}_{1}-\mathrm{S}_{2}$ step. The changes between $\mathrm{Mn}^{\mathrm{IV}}(\mathrm{sal})_{2}$ (bipy) and $\left[\mathrm{Mn}{ }^{\mathrm{III}}(5-\mathrm{Cl}-\mathrm{Salpn})\left(\mathrm{CH}_{3} \mathrm{OH}\right)_{2}\right]\left(\mathrm{O}_{3} \mathrm{SCF}_{3}\right)$ thus approximately reproduce the values for $S_{1}$ and $S_{2}$, while the changes between $\mathrm{Mn}^{\mathrm{IV}}$ (sal) ${ }_{2}$ (bipy) and $\mathrm{Mn}^{\mathrm{III}}$ (acac) $)_{3}$ are larger by a factor of 2 .

Ligand Field Multiplet Interpretation. Calculations that aim to simulate inner-shell spectra can be grouped into density functional-based methods (e.g., FEFF) and wave function-based methods. Transition-metal complexes show strong electronelectron (multiplet) interactions in core hole spectra that are at present not accurately included in any density functional-based approach. ${ }^{47}$ To simulate the $\mathrm{K}$ pre-edge spectral shape, we therefore use multiplet theory that is based on atomic wave function calculations but includes a ligand field. ${ }^{65-67}$ In this first qualitative understanding of the $\mathrm{Mn} \mathrm{K}$ pre-edges, we consider the ionic cases of $\mathrm{Mn}^{2+}, \mathrm{Mn}^{3+}$, and $\mathrm{Mn}^{4+}$. We show that multiplet interactions are crucial to describe the $\mathrm{Mn} \mathrm{K}$ pre-edge spectral shape.

Calculations for $1 \mathrm{~s}$ to $3 \mathrm{~d}$ quadrupole transitions for $\mathrm{Mn}^{2+}\left(3 \mathrm{~d}^{5}\right.$ $\left.\rightarrow 1 \mathrm{~s} 3 \mathrm{~d}^{6}\right), \mathrm{Mn}^{3+}\left(3 \mathrm{~d}^{4} \rightarrow 1 \mathrm{~s} 3 \mathrm{~d}^{5}\right)$, and $\mathrm{Mn}^{4+}\left(3 \mathrm{~d}^{3} \rightarrow 1 \mathrm{~s} 3 \mathrm{~d}^{4}\right)$ configurations are shown in Figure 10. An octahedral symmetry was assumed for $3 \mathrm{~d}^{3}$ and $3 \mathrm{~d}^{5}$, with $10 \mathrm{Dq}$ set to 1.1 and $2.2 \mathrm{eV}$, respectively. The $10 \mathrm{Dq}$ value for the $\mathrm{Mn}^{2+}$ calculation was taken from the $\mathrm{MnO}$ spectrum. The other values were obtained by using the simple rule $10 \mathrm{Dq}=\mathrm{Mn}$ oxidation state $* 0.6 \mathrm{eV}$ and then slightly reduced to account for the $1 \mathrm{~s}$ excited state. ${ }^{48} \mathrm{~A}$ Jahn-Teller distortion was included for $3 \mathrm{~d}^{4}$ by using $D_{4 h}$

(65) Slater, J. C. Quantum Theory of Atomic Structure; McGraw-Hill: New York, 1960

(66) Sugano, S.; Tanabe, Y.; Kamimura, H. Multiplets of Transition-Metal Ions in Crystals; Academic Press: New York, 1970.

(67) Thole, B. T.; Cowan, R. D.; Sawatzky, G. A.; Fink, J.; Fuggle, J. C. Phys. Rev. B 1985, 31, 6856.

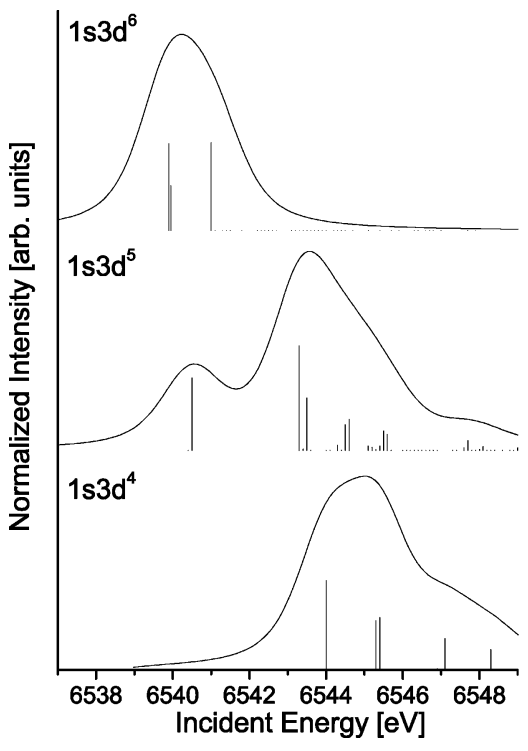

Figure 10. Multiplet calculations for $1 \mathrm{~s}$ to $3 \mathrm{~d}$ quadrupole transitions to $1 \mathrm{~s} 3 \mathrm{~d}^{6}\left(\mathrm{Mn}^{2+}\right), 1 \mathrm{~s} 3 \mathrm{~d}^{5}\left(\mathrm{Mn}^{3+}\right)$, and $1 \mathrm{~s} 3 \mathrm{~d}^{4}\left(\mathrm{Mn}^{4+}\right)$ final states, respectively.

symmetry with $10 \mathrm{Dq}=1.6 \mathrm{eV}$ and Ds set to $0.2 \mathrm{eV}$. The JahnTeller distortion only has a small influence on the spectral shape (as long as a high-spin configuration is preserved) and results mainly in a broadening of the high-energy features. The Slater integrals that describe the electron-electron interactions were scaled down to $80 \%$ of their atomic values. ${ }^{68}$ The calculated stick spectrum was convoluted with a $1.1 \mathrm{eV}$ Lorentzian and a $1.2 \mathrm{eV}$ Gaussian line shape to account for lifetime and instrumental broadening, respectively.

The theoretical spectra have to be compared to the experimental CET line plots. The absolute configuration energies in Hartree-Fock self-consistent field calculations are not accurate. ${ }^{69}$ The calculated spectra were therefore shifted such that the peak in the experimental $\mathrm{MnO}$ spectrum corresponds to the calculated $1 \mathrm{~s} 3 \mathrm{~d}^{6}$ excited-state spectrum and the low energy peak in $\mathrm{Mn}_{2} \mathrm{O}_{3}$ corresponds to the low energy peak in the calculated $1 \mathrm{~s} 3 \mathrm{~d}^{5}$ excited-state spectrum. The calculated $1 \mathrm{~s} 3 \mathrm{~d}^{4}$ excited-state spectrum was shifted such that the high energy tail coincides with the experimental $\mathrm{MnO}_{2}$ spectrum.

This simple theoretical approach neglects many effects such as band structure formation, hybridization, and spectral intensity arising from dipole transitions. The neglected mechanisms have to be included to accurately reproduce the spectral intensities. However, to our knowledge there is at present no theoretical model that correctly combines electronic multiplets including spin-orbit interaction and full symmetry mixing in inner-shell spectra with molecular wave functions that are calculated based on an ab initio theory. We will show that crystal field multiplet theory already reproduces some important aspects.

The $\mathrm{Mn}^{2+} 1 \mathrm{~s} 3 \mathrm{~d}^{6}$ excited-state spectrum reproduces the spectral shape of the Mn(II) model compound spectrum. The two strong resonances are separated by the crystal field splitting 10Dq. They are not resolved in the convoluted spectrum because of the $1 \mathrm{~s}$ core hole lifetime broadening. The low energy peak is split by the spin-orbit interaction in the $t_{2 g}$ orbital. All

(68) von dem Borne, A.; Johnson, R. L.; Sonntag, B.; Talkenberg, M.; Verweyen, A.; Wernet, P.; Schulz, J.; Tiedtke, K.; Gerth, C.; Obst, B.; Zimmermann, P.; Hansen, J. E. Phys. Rev. A 2000, 62, 052703/1-12.

(69) Meisel, A.; Leonhardt, G.; Szargan, R. X-ray Spectra and Chemical Binding; Springer-Verlag: New York, 1989; Vol. 37. 
electron-electron multiplet splittings are absent in $\mathrm{Mn}(\mathrm{II})$ because of the ${ }^{6} \mathrm{~A}_{1}$ ground-state symmetry. Both $\mathrm{Mn}$ (II) compounds in the present study are well-described by the multiplet calculations using the experimentally derived crystal field splittings. This means that the Mn(II) compounds form almost pure $1 \mathrm{~s} 3 \mathrm{~d}^{6}$ excited states and thus probably pure $3 \mathrm{~d}^{5}$ ground states. The ground-state sextet spin multiplicity has a strong stabilizing effect on the electronic structure because of the $(3 \mathrm{~d}, 3 \mathrm{~d})$ "exchange" interaction between electrons with parallel spins. ${ }^{66,70}$

The $\mathrm{Mn}^{3+} 1 \mathrm{~s} 3 \mathrm{~d}^{5}$ spectrum shows the two strong features that are also observed for the experimental spectra with Mn oxidation states larger than II. The splitting between the two strong resonances is due to $(3 \mathrm{~d}, 3 \mathrm{~d})$ multiplet interactions that have a magnitude of 3-5 eV. In particular, the exchange energy lowers the energy of the corresponding electronic state relative to the mean energy of the entire multiplet. ${ }^{65,71}$ Hence, even though the center of gravity of the entire pre-edge structure shifts to higher energies for $\mathrm{Mn}^{3+}$, we still observe a structure at the same energy as the peak in $\mathrm{Mn}^{2+}$. This interesting behavior is thus due to the absence of ( $3 \mathrm{~d}, 3 \mathrm{~d})$ multiplet splitting in the $\mathrm{Mn}^{2+}$ spectrum and the strong exchange splitting in $\mathrm{Mn}^{3+}$. The $\mathrm{Mn}^{3+}$ low energy peak is formed by only one resonance state and is therefore sharper than the peak at about the same energy in the $\mathrm{Mn}^{2+}$ spectrum. We observe this sharpening of the low energy peak in the experimental spectra when comparing $\mathrm{MnO}$ with $\mathrm{Mn}_{3} \mathrm{O}_{4}$ and $\mathrm{Mn}_{2} \mathrm{O}_{3}$ as well as $\mathrm{Mn}^{\mathrm{II}}(\mathrm{acac})_{2}\left(\mathrm{H}_{2} \mathrm{O}\right)_{2}$ with $\mathrm{Mn}^{\mathrm{III}}$ $(\mathrm{acac})_{3}$ and $\left[\mathrm{Mn}^{\mathrm{III}}(5-\mathrm{Cl}-\mathrm{Salpn})\left(\mathrm{CH}_{3} \mathrm{OH}\right)_{2}\right]\left(\mathrm{O}_{3} \mathrm{SCF}_{3}\right)$ (cf. Figures 4 and 6).

The double structure is not visible for the $1 \mathrm{~s} 3 \mathrm{~d}^{4}$ excited-state spectrum, which corresponds to an ionic $\mathrm{Mn}^{4+}$ complex with a $3 \mathrm{~d}^{3}$ ground-state configuration. This theoretical spectrum approximately reproduces the experimental $\mathrm{MnO}_{2}$ spectrum except for the low energy shoulder in the experiment that is most likely due to some $\mathrm{Mn}^{3+}$ contribution.

The two strong features in the CET spectra are better separated in the Mn oxides than in the coordination complexes. This can be explained by a reduced magnitude of the $(3 \mathrm{~d}, 3 \mathrm{~d})$ Slater integrals that describe the electron-electron interactions and thus split the pre-edge spectral features. A more covalent electron configuration reduces the electron-electron interactions as has been discussed by de Groot. ${ }^{47}$ The scaling of the Slater integrals only has a small influence on the center of gravity energy of the entire multiplet structure.

\section{Discussion}

We find a relation between oxidation state and first moment position within each of the two groups of model compounds measured in this study: the oxides and the coordination complexes. Poor agreement is observed when comparing models between the groups with identical oxidation states. An exception is $\mathrm{Mn}(\mathrm{II})$. We will now try to reconcile this apparent disagreement. The 1s2p RIXS spectra of the two groups of Mn systems studied here show similar spectral shapes and trends of spectral change upon Mn oxidation. The overall spectral shape of all systems can be understood within a ligand field multiplet picture.

(70) Strictly speaking, there is no exchange energy term for equivalent electrons. Direct and exchange integrals have the same form. The mechanism that lowers the energy, however, is the same as that for nonequivalent electrons.

(71) Cowan, R. D. The Theory of Atomic Structure and Spectra; University of California Press: Berkeley, CA, 1981
This suggests a common origin of the spectra even though the solid-state structures are very different. To form a unified interpretation we first have to abandon the concept of oxidation state as a means to group the RIXS spectra of the models. This is most obvious when comparing $\mathrm{MnO}_{2}$ and $\mathrm{Mn}^{\mathrm{IV}}$ (salicylate) ${ }_{2}$ (bipyridine) that show entirely different spectra. The latter compares much better to $\mathrm{Mn}_{2} \mathrm{O}_{3}$ in terms of the first moment positions. Our concept considers ligand-metal covalency and the fact that $1 \mathrm{~s} 2 \mathrm{p}$ RIXS at the K pre-edge probes the electronic structure that is localized on the metal ion.

The Effective Number of 3d Electrons. The multiplet calculations for ionic $\mathrm{Mn}$ species suggest that a $\mathrm{Mn}^{2+}\left(\left|1 \mathrm{~s} 3 \mathrm{~d}^{6}\right\rangle\right.$ final-state configuration) and a $\mathrm{Mn}^{3+}\left(\left|1 \mathrm{~s} 3 \mathrm{~d}^{5}\right\rangle\right.$ final-state configuration) present the extreme cases for all experimental $\mathrm{Mn}$ spectra shown here except $\mathrm{MnO}_{2}$. Covalency can be included in ligand field multiplet theory by considering ligand-to-metal charge transfer using configuration interaction. ${ }^{72}$ The Mn K preedge configurations can thus be described by a $c_{1}\left|1 s 3 d^{5}\right\rangle+$ $c_{2}\left|1 s 3 d^{6} \underline{L}\right\rangle$ excited-state configuration mixing ( $\underline{L}$ denotes a hole in a ligand orbital) where $c_{1}$ and $c_{2}$ are coefficients describing the magnitude of admixture of the respective configuration. The coefficients $c_{1}$ and $c_{2}$ can be derived in a fit of the experimental spectra. ${ }^{47}$ This is beyond the scope of this article and will be published elsewhere. ${ }^{73}$ A $1 \mathrm{~s} 3 \mathrm{~d}^{4}$ configuration corresponding to the excited state of an ionic $\mathrm{Mn}^{4+}$ system only plays a dominant role for $\mathrm{MnO}_{2}$ and can be neglected for all other systems that we investigated in this study.

It is more illustrative to use an electron density picture that is more accurate in treating covalency but fails to reproduce the multiplet structure and thus the spectral shape. We can expand the electron density in terms of atomic orbitals and then determine their charge (and spin) occupation. We obtain fractional orbital population numbers because valence electrons in covalent systems are shared between several atoms. The concept of using the effective number of electrons localized on a specific atom has been suggested before by numerous authors to explain spectral shapes in core hole spectroscopy. ${ }^{28,30,49} \mathrm{We}$ thus assign an effective number of electrons to the metal ion $3 \mathrm{~d}$ shell in the $1 \mathrm{~s}$ excited state. The value of the effective number of $3 \mathrm{~d}$ electrons $n_{3 \mathrm{~d}}^{\text {eff }}$ is a property of a system that is tested in 1s $2 p$ RIXS spectroscopy at the K pre-edge. In the systems studied here this number is 6 for $\mathrm{Mn}$ (II) in the $1 \mathrm{~s}$ excited (preedge) state and decreases with higher oxidation state. The strong stabilizing effect of the $(3 \mathrm{~d}, 3 \mathrm{~d})$ exchange interaction in the sextet ground state of $\mathrm{Mn}(\mathrm{II})$ yields an almost pure configuration. Comparison between the experimental and calculated spectra suggests that $n_{3 \mathrm{~d}}^{\text {eff }}$ does not significantly go below 5 in the $1 \mathrm{~s}$ excited states of all other systems except $\mathrm{MnO}_{2}$.

The statements that we have made thus far about the electronic structure of $\mathrm{Mn}$ describe the excited state in the presence of a 1s vacancy. Thus, we have to ask whether we can learn about the ground-state electronic properties of a system in $1 \mathrm{~s} 2 \mathrm{p}$ RIXS spectroscopy. In particular, the orbital population $n_{3 \mathrm{~d}}^{\text {eff }}$ is tested in the excited state and not in the ground state. Several theoretical approaches, such as the multiplet calculations that we presented above, take the core hole effect into account

(72) van der Laan, G.; Zaanen, J.; Sawatzky, G. A.; Karnatak, R.; Esteva, J. M. Solid State Commun. 1985, 56, 673-676.

(73) Glatzel, P.; de Groot, F. M. F.; Bergmann, U.; Yano, J.; Visser, H.; Yachandra, V. K.; Cramer, S. P. Submitted for publication. 
to simulate the excited-state spectrum. ${ }^{74-77}$ Ground state and excited state are linked via the transition matrix elements, and an excited-state spectrum thus allows us to draw conclusions about the ground-state properties.

In the present study, we analyze a series of spectra that were obtained by the same inner-shell spectroscopic technique. We arrive at our conclusions from the observation of relative spectral changes, in particular the relative shift of the $1 \mathrm{~s}$ to $3 \mathrm{~d}$ excitation energy. The 1 s core hole potential $U_{1 \mathrm{~s} 3 \mathrm{~d}}$ that acts on the valence electrons is to a good approximation equal for all systems; i.e., the core hole affects the valence electrons in the same fashion. Furthermore, the Coulomb potential of the Mn 1s core hole that acts on the ligand electrons is screened by the resonantly excited electron in the Mn 3d shell. Experimental evidence for a similar core hole screening has been found in L-edge spectroscopy. ${ }^{47}$ We can thus transfer our results concerning relative spectral changes to the electron configuration in the ground state. We note, however, that comparison between different spectroscopic techniques requires a careful consideration of the electronic transitions and relaxation processes after electronic excitations and the electron properties that are tested.

We can assume that the Mn K pre-edge structure mainly reflects the value for $n_{3 \mathrm{~d}}^{\text {eff }}$ in all systems. The pre-edge is affected by $n_{3 \mathrm{~d}}^{\text {eff }}$ in two ways: (1) A smaller electron density in the $\mathrm{Mn} 3 \mathrm{~d}$ orbitals reduces the nuclear screening and the preedge center of gravity moves to higher incident energies. (2) The multiplet splittings are distinctly different for $1 \mathrm{~s} 3 \mathrm{~d}^{6}, 1 \mathrm{~s} 3 \mathrm{~d}^{5}$, and $1 \mathrm{~s} 3 \mathrm{~d}^{4}$ configurations. In most real systems, one observes a mixing of the configurations and thus of the spectral shapes. An exception is $\mathrm{Mn}(\mathrm{II})$, which exhibits an almost pure $1 \mathrm{~s} 3 \mathrm{~d}^{6}$ excited-state configuration.

A 1s2p RIXS spectrum in a complex where Mn occurs in different chemical forms, such as $\mathrm{Mn}_{3} \mathrm{O}_{4}$ or PS II, represents a superposition of all spectra arising from the different chemical forms of $\mathrm{Mn}$ in the sample. Our analysis in terms of the effective number of $3 \mathrm{~d}$ electrons therefore describes an average of the $\mathrm{Mn}$ atoms in the tetranuclear cluster. Thus, within this basic analysis where we reduce the spectra to one number we can compare mononuclear complexes and the OEC in PS II.

The first moments in the RIXS plane for all systems are plotted in Figure 11. We obtain a relative ordering along the incident energy axis of all Mn systems studied here in terms of $n_{3 \mathrm{~d}}^{\text {eff }}$, where a larger incident energy means a smaller value for $n_{3 \mathrm{~d}}^{\text {eff }}$. We thus find that $n_{3 \mathrm{~d}}^{\text {eff }}$ for $\mathrm{Mn}$ in all coordination complexes as well as in PS II is smaller than that in $\mathrm{Mn}_{2} \mathrm{O}_{3}$.

Using the orbital population $n_{3 \mathrm{~d}}^{\text {eff }}$, we can define a Mn oxidation as any decrease of the effective number of Mn 3d electrons. The changes per oxidation state in the first moment positions are more pronounced between the Mn oxides than those between the Mn coordination complexes. This observation is consistent with a stronger covalency in the coordination complexes. We furthermore observe a Mn oxidation between the $S_{1}$ and $S_{2}$ states of PS II even though the change in the Mn electronic structure is less pronounced than that in the oxides. The spectral change per $\mathrm{Mn}$ ion between $\mathrm{Mn}^{\mathrm{III}}(\mathrm{acac})_{3}$ and $\mathrm{Mn}^{\mathrm{IV}}$

(74) van der Laan, G.; Zaanen, J.; Sawatzky, G. A.; Karnatak, R.; Esteva, J. M. Phys. Rev. B 1986, 33, 4253-4263.

(75) Rehr, J. J. J. Phys.: Condens. Matter 2003, 15, S647-S654.

(76) Taillefumier, M.; Cabaret, D.; Flank, A. M.; Mauri, F. Phys. Rev. B 2002, $66,195107$.

(77) Shirley, E. L. Phys. Rev. Lett. 1998, 80, 794-797.



Figure 11. First moment positions in the 1s $2 p$ RIXS plane for the Mn oxides ( $(\mathbf{)})$, the coordination complexes $(\bigcirc)$, and PS II (A). A linear fit is shown for the oxides. Along the incident energy axis the plot gives an ordering of the systems in terms of the effective number of $3 \mathrm{~d}$ electrons.

(salicylate) $)_{2}$ (bipy) is by a factor of 2 more pronounced than that between $S_{1}$ and $S_{2}$. In other words, the orbital population change $\Delta n_{3 \mathrm{~d}}^{\text {eff }}$ per change in oxidation state is largest between the $\mathrm{Mn}$ oxides and smallest between $S_{1}$ and $S_{2}$. The reason is an increased covalency or delocalization of the Mn valence orbitals. We thus find that the electron that is transferred from the OEC in PS II between $S_{1}$ and $S_{2}$ is strongly delocalized and cannot be assigned to just one element in the OEC.

The incident energy first moment position of $\left[\mathrm{Mn}^{\mathrm{III}}(5-\mathrm{Cl}-\right.$ Salpn $\left.)\left(\mathrm{CH}_{3} \mathrm{OH}\right)_{2}\right]\left(\mathrm{O}_{3} \mathrm{SCF}_{3}\right)$ is higher than that for $\mathrm{Mn}^{\mathrm{III}}(\mathrm{acac})_{3}$, indicating a lower value for $n_{3 \mathrm{~d}}^{\text {eff }}$ in the former. The positive charge in the inner-sphere complex $\left[\mathrm{Mn}^{\mathrm{III}}(5-\mathrm{Cl}-\mathrm{Salpn})\left(\mathrm{CH}_{3}-\right.\right.$ $\left.\mathrm{OH})_{2}\right]^{+}$is countered by $\left(\mathrm{O}_{3} \mathrm{SCF}_{3}\right)^{-}$without a covalent bond to the metal ion. $\mathrm{Mn}$ in $\left[\mathrm{Mn}{ }^{\mathrm{III}}(5-\mathrm{Cl}-\mathrm{Salpn})\left(\mathrm{CH}_{3} \mathrm{OH}\right)_{2}\right]\left(\mathrm{O}_{3} \mathrm{SCF}_{3}\right)$ therefore appears more "ionic" or "oxidized" than that in $\mathrm{Mn}^{\mathrm{III}}$ $(\mathrm{acac})_{3}$. The magnitude of the spectral change per $\mathrm{Mn}$ ion between $\mathrm{Mn}^{\mathrm{IV}}(\mathrm{sal})_{2}$ (bipy) and $\left[\mathrm{Mn}^{\mathrm{III}}(5-\mathrm{Cl}-\mathrm{Salpn})\left(\mathrm{CH}_{3} \mathrm{OH}\right)_{2}\right]\left(\mathrm{O}_{3}\right.$ $\mathrm{SCF}_{3}$ ) approximately reproduces the $\mathrm{S}_{1}-\mathrm{S}_{2}$ change in PS II, and the incident energy first moment position of $\left[\mathrm{Mn}^{\mathrm{III}}(5-\mathrm{Cl}-\right.$ Salpn $\left.)\left(\mathrm{CH}_{3} \mathrm{OH}\right)_{2}\right]\left(\mathrm{O}_{3} \mathrm{SCF}_{3}\right)$ is equal to the value of the $\mathrm{S}_{1}$ state. The two coordination complexes are therefore good models for the Mn $n_{3 \mathrm{~d}}^{\text {eff }}$ value in PS II and the change $\Delta n_{3 \mathrm{~d}}^{\text {eff }}$ between $\mathrm{S}_{1}$ and $\mathrm{S}_{2}$.

It is important to note that electron density and molecular structure are interdependent. A change in bonding angles and/ or interatomic distances has to be accompanied by a change in electron density. The effective number of Mn $3 \mathrm{~d}$ electrons $n_{3 \mathrm{~d}}^{\text {eff }}$ reflects one property of the electron distribution in the complex and can therefore by affected by a structural change. It was found in EXAFS studies that the overall structure is almost invariant in the $S_{1}$ to $S_{2}$ transition. ${ }^{8}$ We thus attribute the change in $n_{3 \mathrm{~d}}^{\text {eff }}$ to the transfer of an electron from the OEC to the reaction center in PS II.

We can relate density functional theory calculations to our experimental results. The effective number of valence electrons can be derived from the electron density by means of a population analysis. ${ }^{32}$ High-accuracy quantum chemical calculation as they are done by Siegbahn and co-workers, for example, can thus directly be compared to the Mn K pre-edge experimental results. ${ }^{7}$ In practice, calculations and RIXS experiments could be performed on a series of model compounds with well-known structure. The theoretical effective number of $3 \mathrm{~d}$ electrons can be used to obtain a relation between the first moment position on the incident energy axis and the effec- 


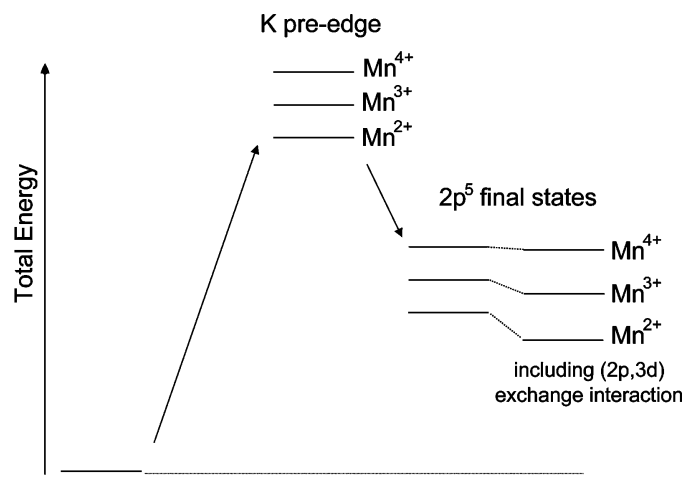

Figure 12. Simplified energy scheme to illustrate the different chemical shifts observed in the K pre-edge and in the $2 \mathrm{p}^{5}$ final states in 1s2p RIXS spectroscopy. The total energy positions of the final states are shown without and with $(2 \mathrm{p}, 3 \mathrm{~d})$ exchange interaction.

tive number of $3 \mathrm{~d}$ electrons. One can then in turn obtain an experimental number of effective $3 d$ electrons for Mn in PS II and use this number as a guide for theoretical calculations.

The Energy Transfer Direction. An interpretation of the first moment shifts in the energy transfer direction is more complicated because of final-state interactions. Multiplet analyses of transition-metal L-edges as well as $\mathrm{K} \alpha$ fluorescence spectra show the importance of the $(2 \mathrm{p}, 3 \mathrm{~d})$ two-electron Slater integrals. ${ }^{35,47} \mathrm{~A}$ shift in the energy transfer direction at the same incident energy, as can be observed for the coordination complexes relative to the $\mathrm{Mn}$ oxides, indicates different finalstate interactions. They introduce an additional chemical sensitivity. We applied a linear fit to the moment positions of the Mn oxides (Figure 11) and found for the slope a value of 1.4. The slope of the fit should be unity if the final-state interactions were zero or equal for all Mn oxides.

The fact that the Mn oxides lie on a straight line in Figure 11 indicates that the magnitude of the final-state interactions changes proportionally to the effective number of $3 \mathrm{~d}$ electrons that causes the shift in the incident energy direction. In other words, the same change in the electronic structure affects the incident energy first moment shift and the final-state interactions.

We propose a model to explain the larger chemical shifts in the energy transfer direction compared to the incident energy shifts. Part of the $(2 \mathrm{p}, 3 \mathrm{~d})$ electron-electron interaction is the exchange energy for electrons with parallel spins. The exchange energy lowers the total energy of these final states. The magnitude of the exchange energy is largest for $\mathrm{Mn}(\mathrm{II})$ and decreases with increasing oxidation state following the number of unpaired electron spins in the valence orbitals. The $\mathrm{Mn}$ (II) final states are therefore lowered most, and the $\mathrm{Mn}(\mathrm{IV})$ final states are lowered least by the $(2 \mathrm{p}, 3 \mathrm{~d})$ exchange interaction. This effect adds to the change in total energy due to the loss in nuclear charge screening, and the shifts are therefore larger in direction of the energy transfer than in direction of the incident energy. The proposed mechanism is illustrated in Figure 12. ${ }^{78}$ The effective number of $3 \mathrm{~d}$ electrons is directly connected to the net spin in the valence shell (effective number of unpaired

(78) The exchange interaction does not change the "center of gravity" of the entire multiplet, but it stretches a spectrum over a larger energy range. By just looking at the $20 \%$ first moment of the " $\mathrm{L}_{3}$ " final states, we select the part of the spectrum that is shifted toward lower total energies by the exchange interaction. 3d electrons). This explains why the Mn oxides lie on a straight line in Figure 11.

Dipole Transitions. A possible concern in our analysis is the influence of dipole contributions in $T_{1}$ (cf. eq 1 ) to the spectra depending on the Mn site symmetry. These will affect the spectral shape and possibly the first moment position. It has been shown for Fe compounds that a change in symmetry with an increase in dipole contribution hardly changes the incident energy pre-edge position. ${ }^{20}$ Furthermore, the $\mathrm{Mn}_{3} \mathrm{O}_{4}$ spectrum with some contribution from the $T_{d}$ site fits into the series of oxides with $O_{h}$ and $D_{4 h}$ symmetry, indicating that dipole contributions in this case only have a small influence on the moment analysis. We can thus use the incident energy first moment for comparison between model compounds and PS II to determine the effective number of $3 \mathrm{~d}$ electrons.

\section{Summary and Conclusions}

An analysis of the $\mathrm{Mn} \mathrm{K}$ pre-edge structure in model compounds and in the $S_{1}$ and $S_{2}$ states of PS II using resonant inelastic X-ray scattering was carried out. The pre-edge absorption spectral shape of all systems can be understood qualitatively within a simple ligand field multiplet picture for 1 s to $3 \mathrm{~d}$ quadrupole transitions. On the basis of the common origin of the spectra, we propose to relate the pre-edge energy position to the effective number of Mn 3d electrons and use this number as a link to quantum chemical calculations.

The spectral changes between the $S_{1}$ and $S_{2}$ states of PS II are not consistent with the removal of an electron from one of the four Mn atoms in the OEC. The electron that is transferred from the OEC to the PS II reaction center thus occupies a strongly delocalized orbital in the OEC. It cannot be assigned to just one element. This contradicts the notion of a localized change of electron configuration, i.e., a localized oxidation. We note that our results are not in disagreement with results from other spectroscopic techniques. Magnetic resonance spectroscopies, for example, are sensitive to the spin of the delocalized electron density and therefore do not test the local Mn electron configurations.

The energy transfer final states are sensitive to the valence shell spin state via the $(2 \mathrm{p}, 3 \mathrm{~d})$ multiplet interaction. The energy transfer spectral resolution is only limited by the $2 p$ core hole lifetime of $0.5 \mathrm{eV}$. The total instrumental energy broadening of presently $1.2 \mathrm{eV}$ can be reduced with some effort to below 0.6 $\mathrm{eV}$ with an acceptable loss in incident photon flux. This will allow for a more detailed analysis and give new information on the $(2 \mathrm{p}, 3 \mathrm{~d})$ multiplet interactions and thus the Mn spin state.

The model systems investigated here do not reproduce the PS II results in the energy transfer direction. It is desirable for future studies to include tetranuclear model systems that reproduce the RIXS spectral shape in PS II and the moment positions in the incident energy as well as energy transfer direction. Furthermore, a 1s2p RIXS study of all stable S-states $\left(\mathrm{S}_{0}-\mathrm{S}_{3}\right)$ in connection with density functional calculations promises to give deeper insight into the electronic structure of Mn in PS II.

Acknowledgment. This work was supported by the National Institutes of Health (Grant GM55302 (V.K.Y.) and GM-65440 (S.P.C.)), National Science Foundation under Grant No. 0213592 (S.P.C.) and the Director, Office of Science, Office of Basic 
Energy Sciences, Division of Chemical Sciences, Geosciences, and Biosciences, of the U.S. Department of Energy (DOE), under Contract DE-AC03-76SF00098 (V.K.Y.) and DOE Office of Biological and Environmental Research (S.P.C.). Use of the Advanced Photon Source was supported by the U.S. Department of Energy, Basic Energy Sciences, Office of Science, under Contract No. W-31-109-ENG-38. BioCAT is a National Institutes of Health-supported research center RR-08630. We thank
Prof. K. Sauer (University of California at Berkeley) for insightful discussions and his encouragement.

Supporting Information Available: Description of fitting procedure to subtract the $\mathrm{K}$ main edge. This material is available free of charge via the Internet at http://pubs.acs.org.

JA038579Z 